\title{
THE LIFE AND WORK OF ROBERT J. ADCOCK. PART I. THE FIGURE OF THE EARTH
}

\author{
by
}

\section{WOLFGANG H. MÜLLER ${ }^{1 *}$ and RICHARD J. HOWARTH ${ }^{2}$}

\author{
${ }^{1}$ Technische Universität Berlin, Fak. V, Institut für Mechanik, Lehrstuhl für Kontinuumsmechanik und \\ Materialtheorie, Sekr. MS 2, Einsteinufer 5, D-10587 Berlin, Germany. \\ ${ }^{2}$ UCL Earth Sciences, 5 Gower Place, London WC1E 6BS, UK. \\ *whmueller1000@gmail.com; r.howarth@ucl.ac.uk.
}

The American mathematician Robert Jackson Adcock (1826-1895) is an obscure figure, hitherto associated with the history of regression analysis and least-squares, whose identity and life is described in Part II of this work. In 1872, he self-published a pamphlet, Gravitation to the sphere and the two ellipsoids of revolution: ratio of the axes of a rotating fluid mass, which seems to have been largely ignored at the time. It effectively became lost thereafter, until a copy was recently discovered in the Library of the Royal Society. In it, he determined the degree of flattening assumed by a rotating homogeneous ellipsoidal fluid Earth with a uniform density, subject to gravitational attraction and in hydrostatic equilibrium; a problem previously considered, but not solved, by Newton, Laplace, Gauss and Dirichlet. Adcock successfully obtained an explicit solution for the potential of a homogeneous ellipsoid and correctly calculated the flattening of such a model. Until now, this result was believed to have first been obtained by a German engineer, Otto Heymann in 1935. Adcock's pamphlet is transcribed here with a commentary on its contents. It is hoped that recognition of his remarkable achievement will enhance his reputation as a mathematician.

Keywords: Figure of the earth; dynamics; potential theory; physical astronomy; history of mathematics.

\section{INTRODUCTION}

For many years, the name of the American mathematician, 'R. J. Adcock,' has been associated with the history of regression, specifically, fitting a straight-line relationship, $y=\alpha+\beta x$, when both $x$ and $y$ are subject to error, and ideas associated with the eventual development of principal components analysis. ${ }^{1}$ An account of his life, so far as its details can now be ascertained, is given in Part II of this work.

The majority of his mathematical publications were short notes, mathematical conundrums, or solutions to his own problems or to those set by others. These appeared mainly in the journal The Analyst (1874-1883), but he also published in The Annals of Mathematics, The American Mathematical Monthly, The Astronomical Journal and The Sidereal Messenger. Unfortunately, his contributions, including the self-published work discussed here, were sometimes marred by algebraic or arithmetic errors, both of his own making ${ }^{2}$ or attributed to the compositor. ${ }^{3}$ 


\section{GRAVITATIONAL ATTRACTION AND THE FIGURE OF THE EARTH}

In the late 1600s, the English scientist Robert Hooke (1635-1703) was thinking about the orbital dynamics of planetary motions and concluded that:

All Coelestial Bodies whatsoever, have an attraction or gravitating power towards their own Centres, whereby they attract not only their own parts, and keep them from flying from them, as we may observe the Earth to do, but that they do also attract all the other Coelestial bodies that are within the sphere of their activity. ${ }^{4}$

Similar ideas had been considered by earlier investigators, including the French astronomer, Ismaël Bullialdus (1605-1694), the Italian physicist, Giovanni Borelli (1608- 1679), and by Hooke's fellow-countryman, Isaac Newton (1643-1727), but it was Hooke's suggestion

that all bodies whatsoever that are put into a direct and simple motion, will so continue to move forward in a streight line, till they are by some other effectual powers deflected and bent into a Motion, describing a Circle, Ellipsis, or some other more compounded Curve Line. ${ }^{5}$

which he applied to planetary motion around the Sun, believing that gravitational attraction originated in periodic pulses (analogous to light and sound). His notion of compounding the tangential velocity of a body with a radial velocity resulting from its attraction to a central body (such as the Sun), proved immensely helpful to Newton's consideration of the mechanisms of celestial dynamics. In a subsequent letter to Newton in January 1679, Hooke revealed that he supposed that 'the Attraction always is in a duplicate proportion to the Distance from the Centre Reciprocall,' ${ }^{6}$ i.e. that the force of gravity is inversely proportional to the square of distance. By so-doing he precipitated a priority dispute with Newton who had arrived at the same idea independently, based on a sound theoretical basis, which Hooke lacked, by 1669, but had not published it. ${ }^{7}$ However, in Newton's Philosophiae naturalis principia mathematica (Mathematical principles of natural philosophy), in Proposition LXXVI, Theorem XXXVI, he states:

\footnotetext{
If spheres are in any way nonhomogeneous (as to the density of their matter and their attractive force) going from the center to the circumference, but are uniform throughout in every spherical shell at any given distance from the center, and the attractive force of each point decreases in the squared ratio of the distance of the attracted body, I say that the total force by which one sphere of this sort attracts another is inversely proportional to the square of the distance between their centres. ... Hence, if many spheres of this sort, similar to one another in all respects, attract one another, the accelerative attraction of any one to any other of them, at any equal distances between the centres, will be as the attracting spheres. And at any unequal distances, as the attracting sphere divided by the square of the distances between the centers. ${ }^{8}$
}

As shown by Chandrasekhar, ${ }^{9}$ later in this Proposition, Newton proves that the (gravitational) attractive force $F$ between any pair of spheres $i$ and $j$, with masses $M_{i}$ and $M_{j}$, separated by a distance $R_{i j}$ is:

$$
F\left(M_{i}, M_{j}\right)=\mathcal{C} \frac{M_{i} M_{j}}{R_{i j}^{2}}
$$

where $\mathcal{C}$ is a constant of proportionality (which has since come to be known as Newton's gravitational constant). Ideally, if $M_{\oplus}$ is the mass of the Earth; $R_{\oplus}$ is its mean radius; the mean gravitational acceleration at the Earth's surface is $g$; and its mean density is $\rho_{\oplus}$; then:

$$
\mathcal{C}=\frac{g M_{\oplus}}{R_{\oplus}^{2}}=\frac{3 g}{4 \pi R_{\oplus} \rho_{\oplus}}
$$


Although Newton had no ready means of determining its value, he foresaw that it ought to be possible to do so experimentally. However, subsequent investigators initially focused on $\rho_{\oplus}$. The first of these determinations was attempted by the French mathematician, geophysicist, geodesist, and astronomer, Pierre Bouguer (1698-1758) and the geographer and mathematician, Charles Marie de la Condamine (1701-1744) at Chimborazo in Peru in 173740: If $g_{h}$ is the value of gravity measured on a plateau of height $h$ above sea level, compared with a value of $g_{0}$ at sea level, and $d$ is the density of the plateau then, ideally:

$$
g_{h}=g_{0}\left(1-\frac{2 h}{R_{\oplus}}+\frac{3 h d}{R_{\oplus} \rho_{\oplus}}\right)
$$

The rocks forming the plateau provide $d ; R_{\oplus}$ is determined from astronomical observations, and hence $\rho_{\oplus}$ may, in theory, be found. Unfortunately, their pendulum-based gravity measurements gave inconclusive results. ${ }^{10}$

The next attempt to measure the 'attraction of a mountain' was made at Schiehallion in Scotland in 1744-6 by the Astronomer Royal, Neville Maskelyne (1732-1811) and mathematician, Charles Hutton (1737-1823), using a plumb-line. ${ }^{11}$ This gave a mean density of 4.5-5 $\mathrm{g} \mathrm{cm}^{-3}$. Similar results followed, but in 1797-8, the English natural philosopher Henry Cavendish (1731-1810), using a torsion balance newly invented by the Rev. John Michell (1724-1793; who unfortunately died before he could use it), found the Earth's density to be $5.448 \mathrm{~g} \mathrm{~cm}^{-3}$ which corresponds to $\mathcal{C}=6.74 \times 10^{-11} \mathrm{~m}^{3} \mathrm{~kg}^{-1} \mathrm{~s}^{-2} .{ }^{12}$ The superiority in precision achievable by this approach over pendulum measurements was evident and improved instruments of this type have continued to be used into modern times. ${ }^{13}$ The present-day designation of the gravitational constant by $G$ (often referred to as 'big $G$ ' to distinguish it from 'little $g$ ') was introduced by Charles Vernon Boys (1855-1944) in 1894, who found its value to be $6.658 \times 10-{ }^{11} \mathrm{~m}^{3} \mathrm{~kg}^{-1} \mathrm{~s}^{-2} \cdot 14,15$

In his discussion of Proposition LXXVI, Newton had shown that for an object in a circular orbit with a mean radius $r$ around the Sun (or a planet) and a period $T$, if the central object is spherically symmetric and has a radius $R$, then the mean density of the object $\bar{\rho}$ is:

$$
\bar{\rho}=k\left(\frac{r}{R}\right)^{3} \frac{1}{T^{2}}
$$

where the constant $k=\frac{3 \pi}{G} \cdot{ }^{16}$ This tended to focus the aim of subsequent investigations on determining the density of the Earth. ${ }^{17}$

However, a second topic of interest had become apparent while Newton was preparing successive editions of the Principia: namely, the shape, or 'figure,' of the Earth. Between January and April 1691, observations of the planet Jupiter by the Italian astronomer Giovanni Cassini (1625-1712) had found its polar diameter to be shorter 'by a fifteenth part' than its equatorial diameter. This was later confirmed by observations by the English astronomer, James Pound (1669-1724) in 1719 using a '123 foot telescope and an excellent micrometer' 18 who found it to be 1/13. ${ }^{19}$ Then, in 1673, the French astronomer Jean Richer (1630-1696) found the length of a pendulum beating seconds at Cayenne $\left(4.9^{\circ} \mathrm{N}\right.$, French Guiana) to be shorter than its previously measured length in Paris $\left(48.9^{\circ} \mathrm{N}\right)$ by a 'ligne $1 / 4^{\prime}{ }^{20}(0.56 \mathrm{~mm}) .^{21}$ The notion of a planet's shape necessarily being that of a perfect sphere was no longer tenable. 
These findings prompted Newton in the second (1713) edition of the Principia $^{22}$ to propose that a homogeneous, once fluid, Earth would achieve a state of hydrostatic equilibrium. ${ }^{23}$ By considering the behaviour of two fluid-filled hypothetical 'canals' or 'columns,' which extended from the centre to the surface of the body along the polar and equatorial semidiameters, and which he assumed to balance or weigh the same; the whole attracting according to the universal inverse-square law, possessing a uniform density, and rotating about its axis of symmetry (i.e. the N-S polar axis); he showed that it would take the form of an oblate ellipsoid whose ellipticity is infinitesimal. Regarding the Earth's mean density, he argued that:

Our globe of earth is of greater density than it would be if the whole consisted of water only, I thus make out. If the whole consisted of water only, whatever was of less density than water, because of its less specific gravity, would emerge and float above. ... The Earth, if it were not for its greater density, would emerge from the seas, and according to its degree of levity, would be raised more or less above their surface. . . By the same argument, the spots of the Sun, which float upon the lucid matter thereof, are lighter than that matter. And however the Planets have been form'd while they were yet in fluid masses, all the heavier matter subsided to the centre. Since therefore the common matter of our Earth on the surface thereof, is about twice as heavy as water, and a little lower, in mines, is found about three or four, or even five times more heavy; it is probable that the quantity of the whole matter of the Earth may be five or six times greater than if it consisted all of water'. ${ }^{24}$

He defined the degree of ellipticity (also known as flattening) as $f=\frac{d_{E}-d_{P}}{d_{E}}$, where $d_{E}$ is the (mean) equatorial semi-diameter and $d_{P}$ is the polar semi-diameter. Letting

$$
\lambda=\left(\frac{\text { centrifugal acceleration at the equator }}{\text { mean gravitational acceleration at the equator }}\right),
$$

he showed that if $g_{E}$ is the acceleration due to gravity at the equator and $g_{P}$ is the acceleration due to gravity at the pole, then:

$$
\frac{g_{P}}{g_{E}}=\frac{d_{E}}{d_{P}}(1-\lambda)=\frac{1-\lambda}{1-f} \cong(1+f-\lambda)
$$

and, for small $f$ :

$$
\frac{g_{P}}{g_{E}}=1+\frac{f}{5}=1+f-\lambda \text {, and so } f=\frac{5}{4} \lambda .
$$

Contemporary measurements had found that 'in the latitude of Paris $\left[48^{\circ} 50^{\prime} 10^{\prime \prime}\right]$ a heavy body falling in a second of time, describes . . 21737 lines' and

a body in every sidereal day of $23^{\text {h }} 56^{\prime} 4^{\prime \prime}$ uniformly revolving in a circle at the distance $[R]$ of 19615800 [Paris] feet from the centre, in one second of time describes an arc $[\ell]$ of 1433.46 feet; the versed sine of which is $\left[\cong \ell^{2} / 2 R\right] 0.05236561$ feet or 7.54064 lines. And therefore the force with which bodies descend in the latitude of Paris is to the centrifugal force of bodies in the equator arising from the diurnal motion of the Earth, as 2174 to $7.54064 .^{25}$

The centrifugal force of bodies in the equator, is to the centrifugal force with which bodies recede directly from the Earth in the latitude of Paris ... in the duplicate proportion of the radius to the cosine of the latitude, that is, as 7.54064 to 3.267 . Add this force to the force with which bodies descend by their weight in the latitude of Paris, and a body in the latitude of Paris, falling by its whole undiminished force of gravity, in the time of one second, will describe 2177.267 lines. . . .And the total force of gravity in that latitude will be to the centrifugal force of bodies in the equator of the Earth, as 2177.267 to 7.54064 , or as 289 to 1 [i.e. $\lambda=\frac{1}{289}$ and hence $\left.f=\frac{1}{230}\right] \cdot{ }^{26}$ 
Unfortunately, because of the abstruse way in which he expressed his arguments, as well as omissions in his explanations, his theory 'struck even the most reputable continental mathematicians of his time as incomprehensible' ${ }^{27}$

Chandrasekhar ${ }^{28}$ and Müller ${ }^{29}$ have reconstructed in some detail what Newton's chain of reasoning must have been. Nevertheless, despite the difficulties met with in understanding his arguments, Newton's conclusion prompted many years of practical investigations, conducted in many parts of the world by the most capable scientists and astronomers of the time, to measure both the length of a $1^{\circ}$ arc of latitude and the force of gravity at different latitudes, so as to determine the correct value of the figure of the Earth. Newton concluded, on the basis of the evidence of the few pendulum measurements available up to 1704, that 'the Earth is a little higher under the equator than by the ... calculus, and a little denser at the centre than in mines near the surface'. ${ }^{30}$ Variable-density models were subsequently considered by Clairaut, Legendre, Maclaurin and Laplace. ${ }^{31}$

Remarkably, by assuming the Earth to be composed of a series of thin shells which gradually became more spherical as one approached the centre, and that its density $(\Delta)$ decreased outwards from the centre as a function of relative distance $(0 \leq d \leq 1), \Delta=\frac{\sin (m d)}{d}$, where $m$ is an empirical constant, Legendre determined that with $\lambda=\frac{1}{288}$ and setting $m=\frac{7 \pi}{8}$, the ellipticity at the surface would be $\frac{1}{319}$ and at the centre $\frac{1}{424}$, giving 'a flattening of the Earth conforming to that which we adopt from pendulum measurements' and a density at the centre approximately seven times that at the surface. ${ }^{32}$

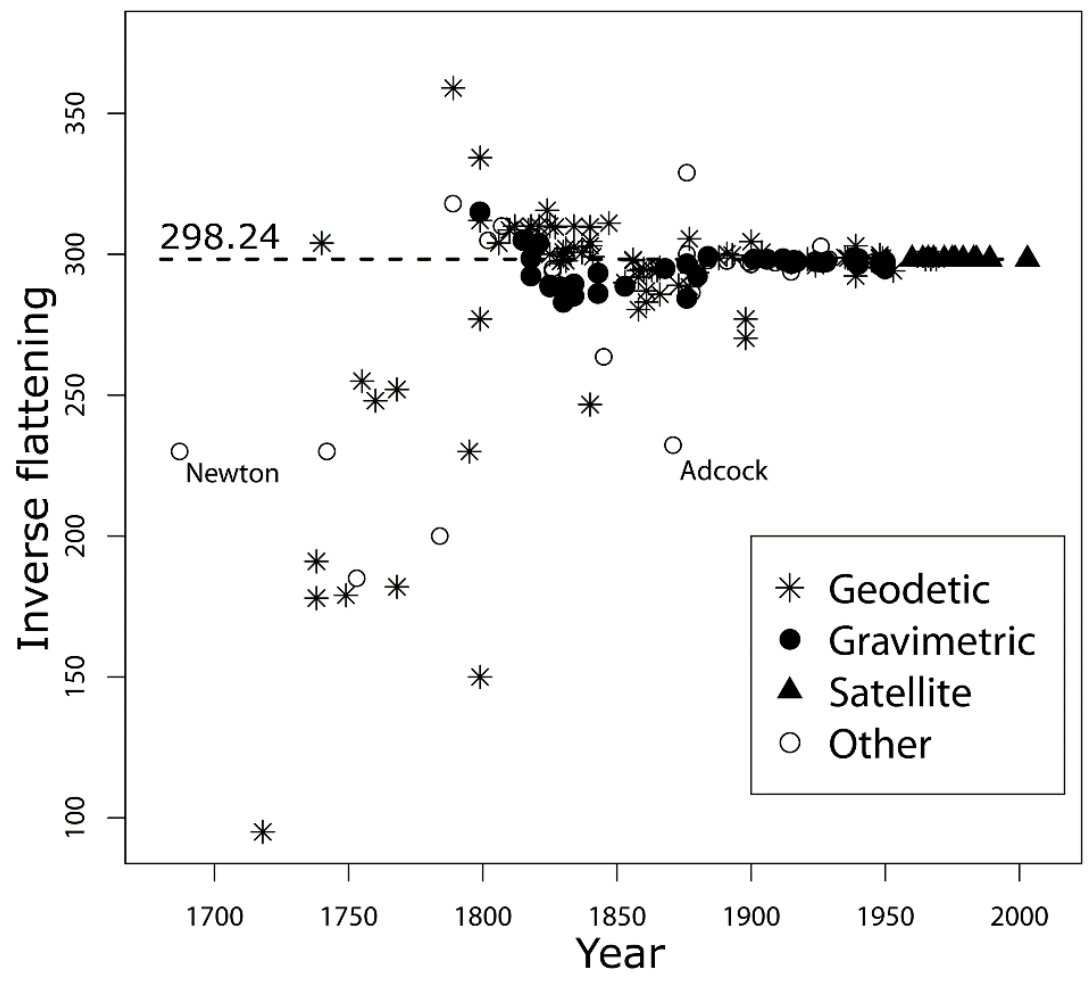

Fig. 1. Determinations of the flattening or 'figure' of the Earth using geodetic, gravimetric (pendulum) and Earth-orbiting satellite measurements ('other' includes purely theoretical calculations) since the time of Newton, expressed as inverse flattening values for convenience. Dashed line shows the currently accepted flattening value of $(1 / 298.24)$.
By the 1870s, numerous publications discussing mathematical theories of attraction and the figure of the earth had appeared and determinations of flattening based on gravimetric measurements were converging on what is now the accepted value (first established in the late 1950s using Earth-orbiting satellites and refined subsequently) of $1 / 298.24$ (Fig. 1). ${ }^{33}$ Pratt's book on the figure of the earth ${ }^{34}$ (with which Adcock was familiar, see below) confirmed that by assuming that the ellipticity of the strata decreased and their density increased as one approached the centre 
of the Earth, and taking a mean density for the Earth of $6.66 \mathrm{~g} \mathrm{~cm}^{-3}$ (based on the result obtained by George Biddell Airy (1801-1892) from experiments at the Harton Pit coalmine in Derbyshire, England), ${ }^{35}$ the estimated flattening was $\frac{1}{307} \cdot{ }^{36}$ Pratt concluded that the discrepancy between Newton's estimate of an inverse flattening of 230 and one of approximately 300 could therefore be explained by having an inhomogeneous Earth whose density increased from the surface to the centre, rather than a homogeneous model. ${ }^{37}$

On $26^{\text {th }}$ August 1872, Adcock gave a paper at the $21^{\text {st }}$ meeting of the American Association for the Advancement of Science (AAAS) at Dubuque, Iowa, titled The Force at any Point of the Surface of a Rotating Fluid Ellipsoid of Three Unequal Axes, under the Action of the Gravity of its own Particles and the Accompanying Centrifugal Force. He seems to have regarded this work as being of major importance, as he had already gone to the expense of paying for a written version, with the title Gravitation to the sphere and the two ellipsoids of revolution: ratio of the axes of a rotating fluid mass, to be printed (see below) and he had sent a copy of it to his local newspaper, the Monmouth Review, Monmouth, Illinois (IL), in the last week of January 1872, as well as having announced it in a monthly church newspaper, the Gospel Echo and Christian, Quincy, IL. ${ }^{38}$ Thereafter, for several months, he advertised it for sale at a price of $\$ 2$ (equivalent to about $\$ 40$ today) ${ }^{39}$ in The Journal of the Franklin Institute and The American Journal of Science and Arts. He also sent copies to the Library of Congress, Washington; Transylvania University, Lexington, Kentucky; Yale University, New Haven, Connecticut; the Washburn Observatory of the University of Wisconsin, Madison, Wisconsin; and to The Royal Society in London, England.

\section{GRAVITATION TO THE SPHERE AND THE TWO ELLIPSOIDS OF REVOLUTION: RATIO OF THE AXES OF A ROTATING FLUID MASS BY R.J. ADCOCK}

The text which follows is a transcription of the quarto-sized pamphlet which Adcock presented to The Royal Society. ${ }^{40}$ Because of the wider page size, the symbol | is used here to denote the end of each line in the original. His paragraph insets, type style, etc. are retained. The final printed page consists of a list of 39 corrections of typographical errata and, for ease of reference, these are given here as footnotes with the attribution RJA. In addition, we note several errors which he appears to have missed. A commentary on his text follows the transcript.

\section{Cover (Page 1)}

\section{GRAVITATION| TO THE| SPHERE AND THE TWO ELLIPSOIDS OF REVOLUTION:| RATIO OF THE AXES| OF A| ROTATING FLUID MASS.|}

BY R.J. ADCOCK.| CINCINNATI, O.:| WILSTACH, BALDWIN \& Co., Printers.| 1872. 


\section{Page 2 (rear of Cover)}

Entered according to act of Congress, A.D. 1871,| BY R.J. ADCOCK,| In the Office of the Librarian of Congress, at Washington, D.C.| ALL RIGHTS ARE RESERVED.|

\section{Bound-in insert following pg. 1}

\section{CIRCULAR.|}

ATTRACTION OF THE SPHERE AND THE TWO| ELLIPSOIDS OF REVOLUTION ; Ratio of the Axes of a Rotating| Fluid Mass under the action of the gravity of its own| Particles. By R.J. ADCOCK.| Printed by Wilstach, Bald-|win \& Co.,| Cincinnati, Ohio.

For a Post Office Order of Two Dollars, drawn on or pay-|able at the Post Office at Galesburg ${ }^{41}$, I propose to send by mail the above work of eight pages quarto, suitable generally| for Professors of Mathematics and advanced Students, in which is $\mid$ published for the first time, a correct solution of the latter prob-|lem which has employed so much thought and expense, by indi-|viduals since the time of Newton.

$$
\text { Address,| R.J. ADCOCK,| Utah, }{ }^{1} \text { Warren Co., Illinois.| }
$$

\section{Page $3^{42}$}

\section{ATTRACTION OF A CONICAL VOLUME.|}

The attraction of matter concentrated to a point, being directly as its mass and inversely as the square of its| distance from the attracted point. Let| point.|

$k_{,}=$the attraction of a unit mass concentrated at the distance of unity from the attracted

$\delta=$ the density of the attracting material.|

$d \theta$ and $r^{2} d \theta=$ the infinitely small surfaces spherical at the distances unity and $r$ from the center $^{43}$ of a sphere.|

Then the attraction of the elementary volume $r^{2} d \theta d r$ for the center of the sphere is|

$\frac{\delta k, r^{2} d \theta d r}{r^{2}}=\delta k, d \theta d r$

which is independent of $r$, the distance of the attracting material from the attracted point.|

\section{INTEGRATING EQUATION (1.)|}

$\int_{0}^{\theta} \int_{r=r_{2}}^{r=r_{1}} \delta k, d \theta d r=\delta^{\prime} k, \theta\left(r_{1}-r_{2}\right), \quad$ (2.) which is the attraction of a conical or pyramidal frustrum for al point at its vertex, and is independent of its distance from the vertex.| 


\section{ATTRACTION OF A SPHERE AND SPHERICAL SHELL. $\left.\right|^{44}$}

Let $\theta=$ the arc at a unit's distance from the center, and the| measure of the angle it subtends at the center of the arc $=$ the angle CBD [Fig. 2].|

$d \theta=$ the angle DBF.

$d u=$ the elementary arc of rotation of the plane $\mathrm{CBD} \mid$ about $\mathrm{BC}$ as an axis. Then|

$d \theta d u$ sine $\theta$ measures the solid angle at $\mathrm{B}$, the vertex of| the pyramidal frustrum, described by

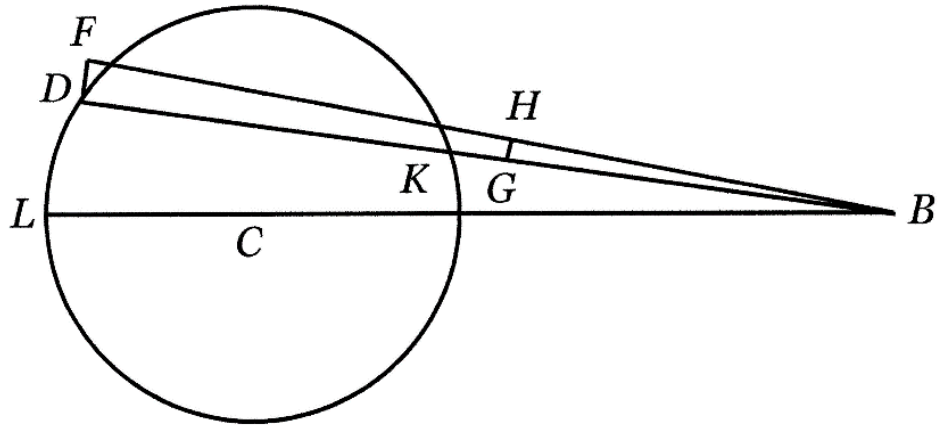

Fig. 2. [This figure had no figure number nor caption in the original manuscript].

DGHF, through the angle| $d u$ about BC. Let|

$\mathrm{DG}=\mathrm{C} e=$ the distance through the attracting material.

By formula (2.) $\delta k, C e$ sine $\theta \cos$. $\theta d \theta d u=$ the component| in the direction $\mathrm{BC}$ of the attraction of the frustrum for the| point $\mathrm{B}$. The double integral of which between the proper|

limits, $\int_{0}^{n} \int_{0}^{\theta} \delta k$, sine $\theta \cos . \theta d \theta d u^{45} \times C e(3 .)^{46}$ is the general expression for the attraction of any geometrical volume| of homogeneous density.|

For the sphere, let $C L=r, C B=a>r$. Then $D K=C e=\left(r^{2}-a^{2} \operatorname{sine} \theta^{2}\right)^{1 / 2}$, and (3.) becomes|

$\int_{0}^{u=2 \pi} \int_{0}^{\theta=\sin ^{\prime} \frac{r}{a}} 2 \delta k_{,}\left(r^{2}-a^{2} \operatorname{sine}^{2} \theta\right)^{1 / 2} \operatorname{sine} \theta \cos . \theta d \theta d u=$

$4 \pi \delta k, \int_{0}^{\theta=\operatorname{sine}^{\prime} \frac{r}{a}}\left(r^{2}-a^{2} \operatorname{sine}^{2} \theta\right)^{1 / 2} \operatorname{sine} \theta \mid \quad \cos . \theta d a=\frac{4 \pi \delta k^{\prime}}{3 a^{2}} 47\left(r^{2}-a^{2} \operatorname{sine}^{2} \theta\right)^{\frac{3}{2}}+$

$C=\frac{4 \pi \delta k_{1} r^{3}}{3 a^{2}}$, the same as its mass $\frac{4}{3} \pi r^{3} \delta$, concentrated in its center.

Differentiating, $d \frac{4}{3} \frac{\pi r^{3} \delta k_{\prime}}{a^{2}}=\frac{4 \pi \delta k^{\prime} r^{2} d r}{a^{2}}=$ the attraction of the spherical shell for the exterior point B.|

And by (1.) the attraction of the shell for an interior point is zero, the attractions of the opposite elementary| frustrums being equal and opposite.

\section{PARTICULAR CASE OF THE ATTRACTION OF THE PROLATE ELLIPSOID| OF REVOLUTION.|}

The distance between the points in which the line $y^{\prime}-y=\tan . \theta\left(x-x^{\prime}\right)$ intersects the ellipse $a^{2} y^{2}+b^{2} x^{2}=a^{2} b^{2}$ is

$C e=\frac{2 a b\left(a^{2} \tan ^{2} \theta+b^{2}-2 x^{\prime} y^{\prime} \tan . \theta-y^{\prime 2}-\tan ^{2} \theta x^{\prime 2}\right)^{1 / 2}\left(1+\tan ^{2} \theta\right)^{1 / 2}}{a^{2} \tan ^{2} \theta+b^{2}}$,

Hence, for the prolate ellipsoid of revolution and exterior point, $\left(x^{\prime}, y^{\prime}\right)$, on longer axis at the distance $h$ from the| center, where $y^{\prime}=0$ and $x^{\prime}=h$, formula (3.) gives

$\int 2 \pi \delta k, C e$ sine $\theta \cos \theta d \theta=\int \frac{4 \pi a b\left(a^{2} \tan ^{2} \theta+b^{2} \cos ^{2} \theta-h^{2} \operatorname{sine}^{2} \theta\right)^{1 / 2} \operatorname{sine} \theta \cos \theta d \theta}{a^{2} \operatorname{sine}^{2} \theta+b^{2} \cos ^{2} \theta}$ 
in which, for the whole ellipsoid, the limits are $\theta=0, \theta=\tan ^{-1} \frac{b}{\left(h^{2}-a^{2}\right)^{1 / 2}}$. Let $\left(a^{2}-h^{2}\right) \operatorname{sine}^{2} \theta+b^{2} \cos ^{2} \theta \mid=z^{2}$, then when $\theta=0, z=b$, and when $\theta=$ $\tan ^{-1} \frac{b}{\left(h^{2}-a^{2}\right)^{2}},{ }^{49} z=0$, and the integral of (5.) is

$$
\left.-\delta k, \int \frac{4 \pi a b z^{2} d z}{b^{2} h^{2}-c^{2} z^{2}}=\frac{4 \pi a^{2} b \delta k_{\prime}}{c^{2}}\left(z-\frac{1}{2} \frac{b h}{c} \log \cdot \frac{b h+c z}{b h-c z}\right)+C, \quad \text { (6. }\right)\left.\right|^{50}
$$

$=\int_{z=0}^{z=b} 2 \pi C_{e} \delta k$, sine $\theta \cos . \theta d \theta=\frac{4 \pi a b^{2} \delta k_{\prime}}{c^{2}}\left(1-\frac{h}{2 c} \log \cdot \frac{h+c}{h-c} \quad\right.$ (7.), 51 which is the attraction of the prolate ellip-|soid of revolution for an exterior point on the major axis, at a distance $h$ from the center.|

Differentiating (7.) with respect $c,{ }^{52} \frac{a}{b}$ and $h$ and $\theta$ being constant|

$$
\delta k, \int_{\theta=\tan .}^{\theta=0} \frac{b}{\left(h^{2}-a^{2}\right)^{1 / 2}} 2 \pi D c C_{e} d c \text { sine } \theta \cos . \theta d \theta=D c \frac{4 \pi a\left(a^{2}-c^{2}\right) \delta k_{\prime}}{c^{2}}\left(1-\frac{h}{2 c} \log \cdot \frac{h+c}{h-c}\right) d c=
$$

$\frac{4 \pi a b^{2} \delta k, d c}{c\left(h^{2}-c^{2}\right)}$,

which is the attraction of the prolate ellipsoidal shell, whose outer and inner surfaces are similar concentric and simi-|larly placed, for a point at the distance $h$ from the center on the major axis.|

\section{A PARTICULAR CASE OF THE OBLATE ELLIPSOID OF REVOLUTION.|}

For an exterior point on the minor axis of the oblate ellipsoid of revolution, in (4.) for $\theta$, $\pi-\theta^{53}$ must be| substituted, and formula (3.) becomes|

$$
\begin{aligned}
& \int_{\theta=0}^{\theta=\tan ^{-1} \frac{a}{\left(h^{2}-b^{2}\right)^{1 / 2}}} 2 \pi a b \delta k, C_{e} \text { sine } \theta \cos . \theta d \theta=
\end{aligned}
$$

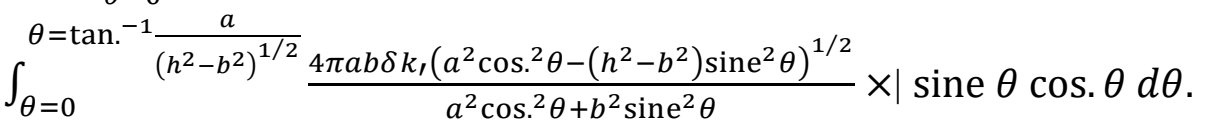

which is the attrac-|tion of the oblate ellipsoid of revolution for an exterior point on its minor axis, at the distance $h$ from the centre.|

Differentiating (10.) with respect to $c$, considering $\frac{b}{a}, \frac{c}{a}, \theta$, and $h$ constant, there results

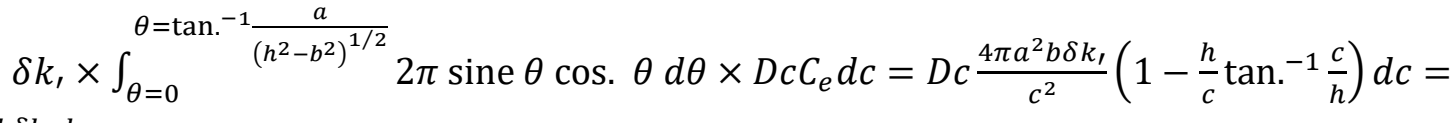
$\frac{4 \pi a^{2} b \delta k, d c}{c\left(h^{2}+c^{2}\right)}, \quad$ (12.)

which is the attraction of the oblate ellipsoidal infinitely thin shell, whose outer and inner surfaces are similar,| concentric and similarly placed, for a point on the minor axis at a distance $h$ from the center.|

$\frac{4 \pi a^{2} b d c}{c}$ is the volume of the shell. Ellipsoidal shells of any thickness having their surfaces so situated, have| their attractions for a point interior to the inner surface zero. For if through the point two opposite infinitessimal ${ }^{54} \mid$ cones be drawn, the distance through the material in the opposite directions are equal, being the semi differences of two| cords, which are the projections of two cords of the two circles of which the elliptic sections are the projections.| And, therefore, by (1.), their attractions are equal. 


\section{DIRECTION OF THE ATTRACTION OF THE ELLIPSOIDAL SHELL.|}

The cord to the ellipse, $C_{e}=\frac{2 a b\left(a^{2} \alpha^{2}+b^{2}+2 x^{\prime} y^{\prime}-y^{\prime 2}-x^{\prime 2} \alpha^{2}\right)^{1 / 2}\left(1+\alpha^{2}\right)^{1 / 2}}{a^{2} \alpha^{2}+b^{2}},(13)^{55}$ in which $\alpha=$ tan. $\stackrel{a}{C_{e}}$ = the| tangent of the angle which $C_{e}$ makes with $a$.|

Differentiating $C_{e}$ with respect to $c ; \frac{a}{b}, \alpha, x^{\prime}, y^{\prime}$, being constant $d C_{e}=$ $\frac{2 a b\left(1+\alpha^{2}\right)^{1 / 2} d c}{c\left(a^{2} \alpha^{2}+b^{2}+2 x^{\prime} y^{\prime}-y^{\prime 2}-x^{\prime 2} \alpha^{2}\right)^{1 / 2}} \cdot{ }^{56}$ (14.)

Put $\alpha=\frac{\beta \mp \delta}{1 \pm \delta \beta}, \beta=\tan .{ }_{a}^{b}=\frac{\alpha_{1}{ }^{2} y^{\prime}}{\left(\alpha^{2}{ }^{2}-c^{2}\right) x}, \delta=\tan .{ }_{C_{e}}{ }_{e}, b_{\prime}=$ the line from $\left(x^{\prime}, y^{\prime}\right)$ to the axis of $x$, bisecting the angle between the two lines from $\left(x^{\prime}, y^{\prime}\right)$ to the foci.|

$a_{1}=$ semi major axis of the ellipse passing through the point $\left(x^{\prime}, y^{\prime}\right)$, and having the same foci as that whose| semi axis is $a$. Then|

$$
\begin{aligned}
& d C_{e}=\frac{2 a b d c}{c}\left(\frac{1+\frac{\beta \mp \delta}{1 \pm \beta \delta}}{\alpha^{2}\left(\frac{\beta \mp \delta}{1 \pm \beta \delta}\right)^{2}+b^{2}+2 x^{\prime} y^{\prime} \frac{\beta \mp \delta}{1 \pm \beta \delta}-y^{\prime 2}-x^{\prime 2}\left(\frac{\beta \mp \delta}{1 \pm \beta \delta}\right)^{2}}\right)^{\frac{1}{2}}=\left.\right|^{57} \\
& \frac{2 a b d c}{c}\left(\frac{\left(1+\beta^{2}\right)\left(1+\delta^{2}\right)}{a^{2} \beta^{2}+a^{2} \delta^{2}+b^{2}+b^{2} \delta^{2} \beta^{2}-2 x^{\prime} y^{\prime} \beta \delta^{2}-y^{\prime 2}-y^{\prime 2} \beta^{2} \delta^{2}-\beta^{2} x^{\prime 2}-x^{\prime 2} \delta^{2}}\right)^{\frac{1}{2}},
\end{aligned}
$$

from which the first power of $s^{58}$ having disappeared, it follows that the differentials of cords on opposite sides of $b_{l} \mid$ making equal angles with it are equal, and, therefore, by (2.) the elementary attractions $\delta k, d C_{e} \operatorname{sine} \theta \cos \theta d \theta$, |

\section{Page 5}

GRAVITATION| [running head]

$\delta k, d C_{e} \operatorname{sine}(-\theta) \cos .(-\theta) d(-\theta)$ are equal, and the same being true for every section through $b$, it must be the direction of the attraction. Therefore, the direction of the attraction of ellipsoidal shells whose outer and inner sur-|faces are similar, concentric, and similarly placed, for an exterior point, is that of the line bisecting the angle| between the lines drawn to the foci on the longest axis of the ellipsoid. And is, therefore, perpendicular to the sur-|face of the ellipsoid passing through the given point $\left(x^{\prime}, y^{\prime}\right)$ and having the same foci as that of the outer surface of| the shell.

\section{ATTRACTION OF SHELLS.|}

Let $d N=$ the infinitely small thickness of a plane lamina. Then $\frac{d N}{\cos . \theta}=C_{e}=$ the distance through it in any| direction $\theta$. And by (3.) $\int_{\frac{1}{2} \pi}^{0} 2 \pi \delta k, \frac{d N}{\cos . \theta} \operatorname{sine} \theta \cos . \theta d \theta=2 \pi \delta k, d N+C=$ $2 \pi \delta k, d N, \quad(16)=$. the attraction of $\mid$ an infinitely thin lamina, infinitely extended, for a point at any distance, $=$ the attraction at distance zero.|

By a formula on page $2,{ }^{59} \frac{4 \pi \delta k, r^{2} d r}{a^{2}}$ being the attraction of a spherical shell at the distance $a$ from the center, $\mid 4 \pi \delta k, d r$ is the attraction for a point on the exterior surface of the shell, and is normal to the surface. The two| parts of a cord between the inner and outer surfaces being equal, $2 \pi \delta k, d r$ is the attraction of all the parts of the| shell at the first or second intersection. Therefore, the attraction of the part at the first intersection of the cord with| any double curved 
lamina, whose two surfaces have a common normal at the point, is $2 \pi \delta k, d r$, because it is evidently| between those of the two spherical lamina of greatest and least curvature, each of which is $2 \pi \delta k, d r, d r=$ the thick-|ness. Hence the attraction, for a point on the exterior surface of any closed shell, of an infinitessimal thickness, | which has the parts of any cord, passing through the given point, between the inner and outer surfaces of the shell,| equal, is $4 \pi \delta k, d N$. That is, $A$ being the attraction of the shell $\frac{A}{d N}=4 \pi \delta k_{\prime}$, (17.) at the limit.|

\section{DIFFERENTIATING UNDER THE SIGN OF INTEGRATION.|}

By the proposition that integrals and differentials are relatively free, it follows that the integral of the differential| coefficient of the quantity under the sign of integration, with respect to any quantity, equals the differential coefficient| of the integral with respect to the same quantity. Giving when $\int d u=\omega$, whether general or definite,

$$
\int D_{h} d u=D_{h} \omega=\frac{d \omega}{d h}, \quad(18 .) \mid
$$

\section{POTENTIAL.|}

Let the term potential $=\omega$, be the general integral of the elementary quantity of work of forces directed to and dependent on the distances to fixed centers from the attracted point.|

A level surface is that which has the potential at every point constant.|

And, consequently, perpendicular to the resultant of the forces at every point, for the elementary quantity of| work, which is the differential of the potential, is zero in no other direction.|

$R=$ resultant of forces, and normal to the level surface.

$d N_{,}=$the distance perpendicular to both, between two level surfaces, infinitely close.|

$d l=\frac{d N_{\prime}}{\cos .{ }_{l}{ }_{l}}=$ the distance between them in the direction $l$. Then $\frac{d \omega}{d N_{\prime}}=R$ (19.), and $\frac{d \omega}{d l}=$ the component of $R$ in the direction $l$.

\section{ATTRACTION OF THE CONCENTRATED ELLIPSOID.|}

Let $a, b, b$, be the semi axes of the ellipsoid, $a$ the greatest and $b$, the least.|

$u=$ the angle which a plane through $b$, makes with $a$.|

$a^{\prime}=$ the major axis of the section. And $a^{\prime 2}-b_{l}^{2}=c_{1}^{2}, \quad$ (20.)

Then $a^{2} a^{\prime 2} \operatorname{sine}^{2} u+b^{2} a^{\prime 2} \cos ^{2} u=a^{2} b^{2}, a^{\prime 2}=\frac{a^{2} b^{2}}{a^{2}-c^{2} \cos ^{2} u}, \quad$ (21.) By (18.) and (12.), $\frac{2 \delta k_{r} d u a^{\prime 2} b_{1} d c_{\prime}}{c_{l}\left(c^{\prime 2}+h^{2}\right)}=\mid$ the attraction of a portion of an ellipsoidal shell for a point on its shorter axis at the distance $h$ from the center,| comprehended between two planes through that axis making the angle $d u$ with each other, and the angle $u$ with| the longest axis.|

Since the outer and inner surfaces are similar, $d c_{,}=\frac{c, d c}{c}$. By (20.), (21.) $c_{l}^{2}=$ $\left.\frac{a^{2} b^{2}-a^{2} b_{1}^{2}+b^{2} c^{2} \cos ^{2} u}{a^{2}-c^{2} \cos ^{2} u} \cdot\right|^{60}$

Then $2 \int_{u=0}^{u=\frac{1}{2} \pi} \frac{2 d u a^{\prime 2} b_{,} d c_{\prime} \delta k_{\prime}}{c_{\prime}\left(c_{I}^{2}+h^{2}\right)}=2 \int_{u=0}^{u=\frac{1}{2} \pi} \frac{2 d u a^{2} b^{2} b_{\prime} d c \delta k_{\prime}}{c\left(a^{2} b^{2}-a^{2} b_{I}^{2}+b_{I}^{2} c^{2} \cos ^{2} u+a^{2} h^{2}-c^{2} h^{2} \cos ^{2} u\right)}=1$ 
$\frac{4 \pi \delta k_{\prime} a b b_{\prime} d c}{c\left(b^{2}-b_{I}^{2}+h^{2}\right)^{\frac{1}{2}}\left(a^{2}-b_{I}^{2}+h^{2}\right)^{1 / 2}}$, in which $c,\left(a^{2}-b_{I}^{2}\right)^{\frac{1}{2}},\left(b^{2}-b_{I}^{2}\right)^{\frac{1}{2}}$ are the semi focal distances of the ellip-|soid, and $\frac{4 \pi a b b, d c}{c}$ is the volume of the shell. Let $\omega=$ the potential of the shell, then by (19.) with respect to $h, \mid \int \frac{4 \pi a b b_{1} \delta k_{1} d c d h}{c\left(b^{2}-b_{I}^{2}+h^{2}\right)^{1 / 2}\left(a^{2}-b_{I}^{2}+h^{2}\right)^{1 / 2}}=\omega$, from which it is evident that shells, of the same foci, whose outer and| inner surfaces are similar and similarly placed concentric ellipsoidal surfaces, have their potentials proportional tol their masses, and, therefore, their attractions for any exterior point in the same ratio, since by proposition, page| $6,{ }^{61}$ and equation (19.), the level surface of the shell is an ellipsoidal surface of the same foci. Let these two ellip-|soids of the same foci be divided into the same number of similar shells, the masses of the shells will be as the masses of the ellipsoids of which they are parts, the component in any direction of the attraction of a shell of one will| be to the component in the same direction of the attraction of the corresponding shell of the other ellipsoid as their| masses, as the masses of the ellipsoids, and, therefore, the sums of these components, or the attractions of the ellipsoids| themselves in any direction, are as their masses. Hence, the attraction of the ellipsoid of homogeneous density| equals the attraction of its mass concentrated into the ellipse whose semi axes are the focal distances $\left(a^{2}-b_{l}^{2}\right)^{1 / 2}, \mid\left(b^{2}-b_{r}^{2}\right)^{1 / 2}$. The density which compressed mass at any point is $\frac{a b b_{1} \delta}{c^{3}}$ multiplied by the double ordinate of the ellipsoid of revolution whose major and minor axes are $2\left(a^{2}-b_{l}^{2}\right)^{1 / 2}, 2\left(b^{2}-b_{l}^{2}\right)^{1 / 2} \cdot \mid$

\section{ATTRACTION OF THE PROLATE ELLIPSOID OF REVOLUTION.}

Since, as just proved, the attractions of ellipsoids of the same foci are as their masses, the attraction of the| prolate ellipsoid of revolution, for an exterior point, equals the attraction of the ellipsoid, of equal mass, whose semi-|axes are the focal distance $c$ and zero. The quantity of matter concentrated on $2 c$ at any point being proportional to $c^{2}-x^{2}, x$ being the distance from the center, and the original density $\delta$ being increased by the ratio of $a b^{2}$ to $c$. Sol that $\frac{a b^{2} \delta}{c}$ being the density at the center, $c^{2}: c^{2}-x^{2}:: \frac{a b^{2} \delta}{c}: \frac{a b^{2} \delta\left(c^{2}-x^{2}\right)}{c^{3}}=$ the density at the point $x$. Hence,| the component, perpendicular to the axis of $x$, of the attraction of the prolate ellipsoid of revolution for an exte-|rior point $\left(x^{\prime}, y^{\prime}\right)$ is

$$
\begin{aligned}
& \frac{\pi a b^{2} \delta k_{\prime}}{c^{3}} \int_{x=-c}^{x=c} \frac{\left(c^{2}-x^{2}\right) y^{\prime} d x}{\left(y^{\prime 2}+\left(x^{\prime}-x\right)^{2}\right)^{\frac{3}{2}}}=\delta k_{\prime}\left(-\frac{\pi a b^{2} y^{\prime}}{c^{3}} \int\left(\frac{\left(c^{2}-x^{\prime 2}\right) d z}{\left(y^{\prime 2}+z^{2}\right)^{\frac{3}{2}}}+\frac{2 x^{\prime} z d z}{\left(y^{\prime 2}+z^{2}\right)^{\frac{3}{2}}}-\frac{z^{2} d z}{\left(y^{\prime 2}+z^{2}\right)^{\frac{3}{2}}}\right)+C \mid\right. \\
= & -\frac{\pi a b^{2} \delta k_{\prime} y^{\prime}}{c^{3}}\left(\frac{\left(c^{2}-x^{\prime 2}\right) z}{y^{\prime 2}\left(y^{\prime 2}+z^{2}\right)^{1 / 2}}-\frac{2 x^{\prime}}{\left(y^{\prime 2}+z^{2}\right)^{1 / 2}}+\frac{z}{\left(y^{\prime 2}+z^{2}\right)^{1 / 2}}-\frac{1}{2} \log \cdot \frac{\left(y^{\prime 2}+z^{2}\right)^{1 / 2}+z}{\left(y^{\prime 2}+z^{2}\right)^{1 / 2}-z}\right)+C=\mid \\
& \frac{\pi a b^{2} \delta k_{\prime} y^{\prime}}{c^{3}}\left(-\frac{\left(c^{2}-x^{\prime 2}\right)\left(x^{\prime}-x\right)}{y^{\prime 2}\left(y^{\prime 2}+\left(x^{\prime}-x\right)^{2}\right)^{1 / 2}}+\frac{x^{\prime}-x}{\left(y^{\prime 2}+\left(x^{\prime}-x\right)^{2}\right)^{1 / 2}}-\frac{1}{2} \log \cdot \frac{\left(y^{\prime 2}+\left(x^{\prime}-x\right)^{2}\right)^{1 / 2}+x^{\prime}-x}{\left(y^{\prime 2}+\left(x^{\prime}-x\right)^{2}\right)^{1 / 2}-x^{\prime}+x}\right)+C \mid \\
= & \frac{\pi a b^{2} \delta k_{\prime}}{c^{3}}\left\{\begin{array}{l}
\frac{\left(x^{\prime 2}-c^{2}\right)\left(x^{\prime}-c\right)}{y^{\prime 2}\left(y^{\prime 2}+\left(x^{\prime}-c\right)^{2}\right)^{1 / 2}}+\frac{x^{\prime}+c}{\left(y^{\prime 2}+\left(x^{\prime}-c\right)^{2}\right)^{1 / 2}}+\frac{1}{2} \log \cdot \frac{\left(y^{\prime 2}+\left(x^{\prime}-c\right)^{2}\right)^{1 / 2}+x^{\prime}-c}{\left(y^{\prime 2}+x^{\prime}-c\right)^{2}-x^{\prime}+c} \\
-\frac{\left(x^{\prime 2}-c^{2}\right)\left(x^{\prime}+c\right)}{y^{\prime 2}\left(y^{\prime 2}+\left(x^{\prime}+c\right)^{2}\right)^{1 / 2}}-\frac{x^{\prime}-c}{\left(y^{\prime 2}+\left(x^{\prime}+c\right)^{2}\right)^{1 / 2}}-\frac{1}{2} \log \cdot \frac{\left(y^{\prime 2}+\left(x^{\prime}+c\right)^{2}\right)^{1 / 2}+x^{\prime}+c}{\left(y^{\prime 2}+\left(x^{\prime}+c\right)^{2}\right)^{1 / 2}-x^{\prime}-c}
\end{array}\right\} \mid 62
\end{aligned}
$$




$$
\begin{aligned}
& \text { Let } y^{\prime 2}=\frac{b_{l}^{2}}{a_{l}^{2}}\left(a_{\prime}^{2}-x^{\prime 2}\right)=\left(a_{1} \pm \frac{c x^{\prime}}{a_{l}}\right)^{2}-\left(x^{\prime} \pm c\right)^{2}=a_{l}^{2}+\frac{c^{2} x^{\prime 2}}{a_{l}^{2}}-x^{\prime 2}-c^{2}, \text { then } \mid \\
& \left(y^{\prime 2}+\left(x^{\prime}+c\right)^{2}\right)^{1 / 2}=a_{\prime}+\frac{c x^{\prime}}{a_{\prime}}, \text { and }\left(y^{\prime 2}+\left(x^{\prime}-c\right)^{2}\right)^{1 / 2}=a_{\prime}-\frac{c x^{\prime}}{a_{\prime}} \text { and } \\
& \frac{1}{2} \log .\left(\frac{\left(y^{\prime 2}+\left(x^{\prime}+c\right)^{2}\right)^{1 / 2}+x^{\prime}+c}{\left(y^{\prime 2}+\left(x^{\prime}+c\right)^{2}\right)^{1 / 2}-x^{\prime}-c} \times \frac{\left(y^{\prime 2}+\left(x^{\prime}-c\right)^{2}\right)^{1 / 2}-x^{\prime}+c}{\left(y^{\prime 2}+\left(x^{\prime}-c\right)^{2}\right)^{1 / 2}+x^{\prime}-c}\right)=\frac{1}{2} \log \cdot\left(\frac{a_{1}+\frac{c x^{\prime}}{a_{\prime}}+x^{\prime}+c}{a_{1}+\frac{c x^{\prime}}{a_{l}}-x^{\prime}-c} \times \frac{a_{1}-\frac{c x^{\prime}}{a_{\prime}}-x^{\prime}+c}{a_{1}-\frac{c x^{\prime}}{a_{\prime}}+x^{\prime}-c}\right) \\
& =\frac{1}{2} \log \cdot \frac{\left(a_{1}+c\right)^{2}-\left(a_{1}+c\right)^{2} \frac{x^{\prime 2}}{a_{l}^{2}}}{\left(a_{1}-c\right)^{2}-\left(a_{1}-c\right)^{2} \frac{x^{\prime 2}}{a_{l}^{2}}}=\log \cdot \frac{a_{1}+c}{a_{I}-c}=2 \log \cdot \frac{c+a_{1}-b_{1}}{c-a_{1}+b_{l}} \text {, so that } \\
& \int_{-c}^{c} \frac{\pi a b^{2} \delta k_{,} y^{\prime}\left(c^{2}-x^{2}\right) d x}{c^{3}\left(y^{\prime 2}+\left(x^{\prime}-x\right)^{2}\right)^{3 / 2}}=\pi a b^{2} \delta k_{\prime} y^{\prime}\left(\frac{2 a_{\prime} c}{b_{l}^{2}}-\log \cdot \frac{a_{\prime}+c}{a_{l}-c}\right)=\text { the component perpendicular to the }
\end{aligned}
$$

\section{Page 7}

\section{GRAVITATION| [running head]}

The component parallel to the same axis|

$$
\begin{aligned}
& =\int \frac{d \omega^{\prime}}{d N_{e}} \times \frac{a_{l}^{2} b_{l}^{2} x^{\prime}}{a_{l}^{2}\left(a_{l}^{4} y^{\prime 2}+b_{l}^{4} x^{\prime 2}\right)^{1 / 2}}=\int_{0}^{c} \frac{4 \pi a b^{2} b_{l}^{2} x^{\prime} d c \delta k_{\prime}}{a_{l} c\left(c^{2} y^{\prime 2}+b_{l}^{4}\right)}=\frac{4 \pi \delta k_{\prime} a b^{2} x^{\prime}}{c^{3}} \int_{a_{l}}^{\left(x^{\prime 2}+y^{\prime 2}\right)^{1 / 2}} \frac{b_{l}^{2} c d a_{\prime}}{a_{l}^{2} y^{\prime 2}} \mid \\
& =\frac{4 \pi a b^{2} \delta k_{\prime} x^{\prime}}{c^{3}} \int_{a_{l}}^{\left(x^{\prime 2}+y^{\prime 2}\right)^{1 / 2}} \frac{\left(a_{l}^{2}-x^{\prime 2}-y^{\prime 2}\right)^{1 / 2} a_{\prime} d a_{\prime}}{\left(a_{\prime}^{2}-x^{2}\right)^{\frac{3}{2}}}=\frac{4 \pi a b^{2} \delta k_{\prime} x^{\prime}}{c^{3}}\left(\frac{\left(a_{l}^{2}-x^{\prime 2}-y^{\prime 2}\right)^{1 / 2}}{\left(a_{l}-x^{\prime 2}\right)^{1 / 2}}\right)-1 \\
& \frac{1}{2} \log \cdot \frac{\left(a_{I}^{2}-x^{\prime 2}\right)^{1 / 2}+\left(a_{1}^{2}-x^{\prime 2}-y^{\prime 2}\right)^{1 / 2}}{\left(a_{I}^{2}-x^{\prime 2}\right)^{1 / 2}+\left(a_{1}^{2}-x^{\prime 2}-y^{\prime 2}\right)^{1 / 2}}=\frac{4 \pi a b^{2} \delta k_{\prime} x^{\prime}}{c^{3}}\left(\frac{c}{a_{I}}-\frac{1}{2} \log \cdot \frac{a_{1}+c}{a_{I}-c}\right) \mid
\end{aligned}
$$

The value of $d \omega^{\prime} \div d N_{e}$ being obtained in the next problem.|

\section{ATTRACTION OF THE OBLATE ELLIPSOID OF REVOLUTION.}

By equations (12.) and (19.)|

$$
d k, \times \frac{4 \pi a^{2} b d c}{c\left(b_{1}^{2}+c^{2}\right)}=\frac{d \omega}{d b_{1}}=\text { the attraction of the oblate shell for the point at the distance } b,
$$

from the center on| the minor axis, $\omega=$ its potential, and $a_{r}^{2} y^{\prime 2}+b_{r}^{2} x^{\prime 2}=a_{r}^{2} b_{r}^{2}$, the equation of a section of its level surface through $\left(x^{\prime}, y^{\prime}\right)$ and the major axis $a_{1}$. Since its level surfaces have the same foci $a_{1} d a_{1}=b_{1} d b_{1}$. Let $d N_{e}=$ the infinitely $\mid$ small perpendicular distance between two level surfaces at $\left(x^{\prime}, y^{\prime}\right) . d N_{e}=\left(1+\frac{d y^{\prime 2}}{d x^{\prime 2}}\right)^{1 / 2} d x^{\prime}=\left(a_{1}^{4} y^{\prime 2}+b_{1}^{4} x^{\prime 2}\right)^{1 / 2} \frac{d x^{\prime}}{d y^{\prime}}=$ $\left.n\right|^{63}$

$$
\begin{aligned}
& \left.d y^{\prime}=\frac{a^{2} y^{\prime}}{b^{2} x^{\prime}} d x^{\prime}=d\left(\frac{b_{\prime}}{a_{l}} a^{\prime 2}-x^{\prime 2}\right)^{1 / 2}\right)^{64}=d \frac{\left(a_{\prime}^{2}-c^{2}\right)^{1 / 2}}{a_{\prime}}\left(a_{\prime}^{2}-x^{\prime 2}\right)^{1 / 2}= \\
& \frac{\left(a_{\prime}^{2}-c^{2}\right)^{1 / 2} a_{\prime} d a_{\prime}}{a_{\prime}\left(a_{\prime}^{2}-x^{\prime 2}\right)^{1 / 2}}+\frac{\left(a_{\prime}^{2}-x^{\prime 2}\right)^{1 / 2} a_{\prime} d a_{\prime}}{\left(a_{\prime}^{2}-c^{2}\right)^{1 / 2} a_{\prime}} \mid \\
& -\frac{\left(a_{I}^{2}-c^{2}\right)^{1 / 2}\left(a_{I}^{2}-x^{\prime 2}\right)^{1 / 2} d a_{\prime}}{a_{I}^{2}}-\frac{\left(a_{I}^{2}-c^{2}\right)^{1 / 2} x^{\prime} d^{\prime}}{a^{\prime}\left(a_{I}^{2}-c^{2}\right)^{1 / 2}}, 65 \frac{a_{I}^{2} y^{\prime}}{b_{I}^{2} x^{\prime}} d x^{\prime}+\frac{b_{I}^{2} x^{\prime}}{a_{l}^{2} y^{\prime}} d x^{\prime}=\frac{y^{\prime} d b_{I}}{b_{I}}+\frac{b_{l}^{3} d b_{I}}{a_{I}^{2} y^{\prime}}- \\
& \frac{b_{1} y^{\prime} d b_{1}}{a_{\prime}^{2}}
\end{aligned}
$$




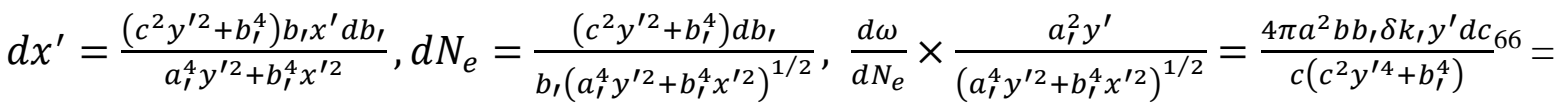
component in| direction $y^{\prime}, \quad b_{l}=\frac{a_{l} y^{\prime}}{\left.a_{l}^{2}-x^{\prime 2}\right)^{1 / 2}},{ }^{67} c=\frac{a_{l}\left(a_{l}^{2}-x^{\prime 2}-y^{\prime 2}\right)^{1 / 2}}{\left(a_{l}^{2}-x^{\prime 2}\right)^{1 / 2}}, \quad a_{l}=\frac{1}{2}\left(y^{\prime 2}+\left(x^{\prime}+\right.\right.$ $\left.c)^{2}\right)^{1 / 2}+\frac{1}{2}\left(y^{\prime 2}+\left(x^{\prime}-c\right)^{2}\right)^{1 / 2}$

$$
\begin{gathered}
d a_{\prime}=\frac{\left(x^{\prime}+c\right) d c}{2\left(y^{\prime 2}+\left(x^{\prime}+c\right)^{2}\right)^{1 / 2}}-\frac{\left(x^{\prime}-c\right) d c}{2\left(y^{\prime 2}+\left(x^{\prime}-c\right)^{2}\right)^{1 / 2}}=\frac{\left(x^{\prime}+c\right) d c}{2\left(a_{1}+\frac{c x^{\prime}}{a_{\prime}}\right)}-\frac{\left(x^{\prime}-c\right) d c}{2\left(a_{,}-\frac{c x^{\prime}}{a_{\prime}}\right)} \\
=\frac{a, c y^{\prime 2} d c}{a_{\prime}^{4}-c^{2} x^{\prime 2}} 68=\frac{a_{1} c y^{\prime 2} d c}{c^{2} y^{\prime 2}+b_{l}^{4}} \cdot \mid
\end{gathered}
$$

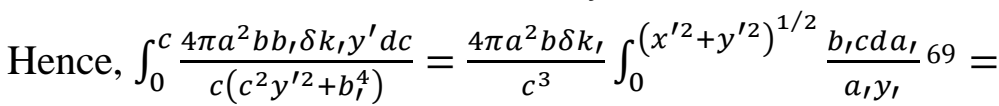
$\frac{4 \pi a^{2} b \delta k_{\prime}}{c^{3}} \int_{0}^{\left(x^{\prime 2}+y^{\prime 2}\right)^{1 / 2}} \frac{a_{\prime} y^{\prime}}{\left(a_{I}^{2}-x^{\prime 2}\right)^{1 / 2}} \times \frac{a_{\prime}\left(a_{l}^{2}-x^{\prime 2}-y^{\prime 2}\right)^{1 / 2}}{\left.a_{l}^{2}-x^{\prime 2}\right)^{1 / 2}} \mid \quad 70 \quad \times \frac{d a_{\prime}}{a_{l} y^{\prime}}=\frac{4 \pi a^{2} b \delta k_{\prime}}{c^{3}} \int \frac{z^{2} d z}{z^{2}+y^{\prime 2}}=$ $\frac{4 \pi a^{2} b \delta k_{\prime}}{c^{3}}\left(z-y^{\prime} \tan ^{-1} \frac{z}{y^{\prime}}\right)=\frac{4 \pi a^{2} b \delta k_{\prime}^{\prime}}{c^{3}}\left(\left(a_{\prime}^{2}-x^{\prime 2}-y^{\prime 2}\right)^{1 / 2}-y^{\prime} \tan ^{-1} \frac{\left(a_{\prime}^{2}-x^{\prime 2}-y^{\prime 2}\right)^{1 / 2}}{y^{\prime}}\right) \mid$ $=\frac{4 \pi a^{2} b \delta k_{1} y^{\prime}}{c^{3}}\left(\frac{c}{b_{l}}-\tan ^{-1} \frac{c}{b_{l}}\right)$, which is the component, perpendicular to the transverse axis, of the attraction of the| oblate ellipsoid of revolution.|

The component parallel to the transeverse ${ }^{71}$ axis equals

$$
\int \frac{d \omega}{d N_{e}} \times \frac{b_{I}^{2} x^{\prime}}{a_{l}^{2}} \times \frac{a_{l}^{2}}{\left(a_{l}^{4} y^{\prime 2}+b_{l}^{4} x^{\prime 2}\right)^{1 / 2}}=\int_{0}^{c} \frac{4 \pi a^{2} b \delta k_{\prime} d c d b_{I}}{a_{\prime}^{2} c} \times \frac{b_{\prime}\left(a_{l}^{4} y^{\prime 2}+b_{l}^{4} x^{\prime 2}\right)^{1 / 2}}{\left(c^{2} y^{\prime 2}+b^{\prime 4}\right)} \times \frac{b_{I}^{2} x^{\prime}}{a^{\prime 2}} \times
$$

$\frac{a_{\prime}^{2}}{\left(a_{\prime}^{4} y^{\prime 2}+b_{l}^{4} x^{\prime 2}\right)^{1 / 2}}$

$=\int_{0}^{c} \frac{4 \pi a^{2} b_{l}^{3} c \delta k_{\prime} x^{\prime} d c}{a_{l}^{2} c\left(c^{2} y^{\prime 2}+b_{l 4}\right)}=\frac{4 \pi a^{2} b \delta k_{\prime} x^{\prime}}{c^{3}} \int_{a_{l}}^{\left(x^{\prime 2}+y^{\prime 2}\right)^{1 / 2}} \frac{b_{l}^{3} c d a_{l}}{a_{l}^{3} y^{\prime 2}}=\frac{4 \pi a^{2} b \delta k_{l} x^{\prime}}{c^{3}} \int_{a_{l}}^{\left(x^{\prime 2}+y^{\prime 2}\right)^{1 / 2}} \frac{a_{l}^{3} y^{\prime 3}}{a_{l}\left(a_{l}^{2}-x^{\prime 2}\right)^{3}} \times$

$\frac{a_{\prime}\left(a_{l}^{2}-x^{\prime 2}-y^{\prime 2}\right)^{1 / 2}}{\left(a_{l}^{2}-x^{\prime 2}\right)^{1 / 2}} \times\left.\frac{d a_{\prime}}{a_{\prime}^{3} y^{\prime 2}}\right|^{72}$

$=\frac{4 \pi a^{2} b \delta k, x^{\prime} y^{\prime}}{c^{3}} \int_{a_{1}}^{\left(x^{\prime 2}+y^{\prime 2}\right)^{1 / 2}} \frac{\left(a_{1}^{2}-x^{\prime 2}-y^{\prime 2}\right)^{1 / 2} a, d a,}{\left(a_{1}^{2}-x^{\prime 2}\right)^{2}}=\frac{4 \pi a^{2} b \delta k, x^{\prime} y^{\prime}}{c^{3}} \int \frac{z^{2} d z}{\left(z^{2}+y^{\prime 2}\right)^{2}}$

$=\frac{4 \pi a^{2} b \delta k_{\prime} x^{\prime} y^{\prime}}{c^{3}}\left(-\frac{z}{2\left(z^{2}+y^{\prime 2}\right)}+\frac{1}{2 y^{\prime}} \tan ^{-1} \frac{z}{y^{\prime}}\right) \mid$

$=\frac{4 \pi a^{2} b \delta k, x^{\prime}}{c^{3}}\left(\frac{1}{2} \tan ^{-1} \frac{\left(a_{\prime}^{2}-x^{\prime 2}-y^{\prime 2}\right)^{1 / 2}}{y^{\prime}}-\frac{\left(a_{\prime}^{2}-x^{\prime 2}-y^{\prime 2}\right)^{1 / 2} y^{\prime}}{2\left(a_{\prime}^{2}-x^{\prime 2}\right)}\right)$

$=\frac{2 \pi a^{2} b \delta k_{\prime} x^{\prime}}{c^{3}}\left(\tan ^{-1} \frac{c}{b,}-\frac{b, c}{a_{r}^{2}}\right) \cdot 1$

In which $a$, and $b$, are the semi axes of the ellipsoid, passing through the attracted point $\left(x^{\prime}, y^{\prime}\right)$, and having the| same foci as that of the given ellipsoid.|

\section{A ROTATING FLUID MASS.}

A fluid mass, in the form of an oblate ellipsoid of revolution, rotating about an axis with the proper angular| velocity, is in equilibrium.

First, when of homogeneous density, let $R=$ the accelerative attraction when at rest, for any point $\left(x^{\prime}, y^{\prime}\right)$, on $\mid$ its meridian section, the latitude of which is $l . \alpha=$ the angular velocity, 
such that $R^{\prime}$, the resultant of $R$ and the| accelerative centrifugal force $\alpha^{2} x^{\prime}$, may be perpendicular to the surface.|

The ellipse gives the equations

$$
\begin{aligned}
& y^{\prime 2}=\frac{b^{2}}{a^{3}}\left(a^{2}-x^{\prime 2}\right) \quad(1 .), \text { sine } l=\operatorname{sine}_{x}{ }_{x}=\operatorname{sine~tan.~}^{-1} \frac{a^{2} y^{\prime}}{b^{2} x^{\prime}}=\frac{a^{2} y^{\prime}}{\left(a^{4} y^{\prime 2}+b^{4} x^{\prime 2}\right)^{1 / 2}} \\
& x^{\prime 2}=\frac{a^{4} \cos ^{2} l}{\left(a^{2} \cos ^{2} l+b^{2} \operatorname{sine}^{2} l\right)}(3 .), \quad y^{\prime 2}=\frac{b^{4} \operatorname{sine}^{2} l}{\left(a^{2} \cos ^{2} l+b^{2} \operatorname{sine}^{2} l\right)}(4 .) \\
& \left(x^{\prime 2}+y^{\prime 2}\right)^{1 / 2}=\left(\frac{\frac{a^{4}}{b^{4}} \cos ^{2} l+\operatorname{sine}^{2} l}{\frac{a^{2}}{b^{2}} \cos ^{2} l+\operatorname{sine}^{2} l}\right)^{\frac{1}{2}}
\end{aligned}
$$

\section{Page 8}

\section{GRAVITATION| [running head]}

$\tan . l=\frac{a^{2} y^{\prime}}{b^{2} x^{\prime}}$. The formula for the attraction are, $\frac{2 \pi a^{2} b \delta k, x^{\prime}}{c^{3}}\left(\tan ^{-1} \frac{c}{b}-\frac{b c}{a^{2}}\right)=R \cos .{ }_{x}^{R}$ $\frac{2 \pi a^{2} b \delta k, y^{\prime}}{c^{3}}\left(\frac{c}{b}-\tan ^{-1} \frac{c}{b}\right)=R \operatorname{sine} . \frac{R}{x}$ (7.), the components perpendicular and parallell ${ }^{75}$ to the axis of rotation.| Since $R^{\prime}$ is the resultant of the two forces $R$ and $\alpha^{2} x^{\prime}$, there results the two equations, $R: R^{\prime}::$ sine ${ }_{x}^{R^{\prime}}:$ sine ${ }_{x}^{R}$ (8.) $\mid R^{\prime 2}=R^{2}+\alpha^{4} x^{\prime 2}-2 \alpha^{2} x^{\prime} R \cos .{ }_{x}{ }^{\prime}$

Hence $R^{\prime}=\frac{R \operatorname{sine} \mathrm{e}_{x}^{R}}{\operatorname{sine} l}=\frac{4 \pi a^{2} b \delta k,\left(\frac{c}{b}-\tan ^{-{ }^{-1}} \frac{b}{b}\right)}{c^{3}\left(a^{2} \cos ^{2} l+b^{2} \operatorname{sine}^{2} l\right)^{1 / 2}}$ (10. $) .{ }^{76} \mathrm{By}$ (8.), (7.), (6.), (2.), (9.), $\quad R^{\prime 2}=$ $\frac{16 \pi^{2} a^{4} b^{2} \delta^{2} k_{\prime}^{2} y^{\prime 2}\left(\frac{c}{b}-\tan ^{-1} \frac{c}{b}\right)^{2}}{c^{6}} \times \frac{a^{4} y^{\prime 2}+b^{4} x^{\prime 2}}{a^{4} y^{\prime 2}}=\frac{4 \pi^{2} a^{4} b^{2} \delta^{2} k_{\prime}^{2} x^{\prime 2}}{c^{6}}\left(\tan ^{-1} \frac{c}{b}-\frac{b c}{a^{2}}\right)^{2}+\left.\right|^{77}$

$\frac{16 \pi^{2} a^{4} b^{2} \delta^{2} k_{\digamma}^{2} y^{\prime 2}}{c^{6}}\left(\frac{c}{b}-\tan ^{-1} \frac{c}{b}\right)^{2}+\alpha^{4} x^{\prime 2}-\frac{4 \pi a^{2} b \delta k, x^{\prime 2}\left(\tan ^{-1} \frac{c}{b}-\frac{b c}{a^{2}}\right)}{c^{3}}$, from which there results, $\left.\right|^{78}$

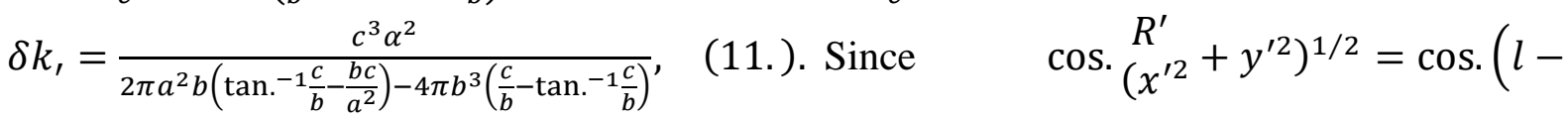
$\left.\tan ^{-1} \frac{y^{\prime}}{x^{\prime}}\right)\left.\right|^{79}=\cos .\left(l-\tan ^{-1} \frac{b^{2}}{a^{2}} \tan . l\right)=\frac{\cos \cdot l}{\left(1+\frac{b^{4}}{a^{4}} \tan \cdot{ }^{2} l\right)^{\frac{1}{2}}}+\frac{\sin \cdot l \times \frac{b^{2}}{a^{2}} \tan ^{2} l}{\left(1+\frac{b^{4}}{a^{4}} \tan ^{2} l\right)^{\frac{1}{2}}} \frac{a^{2} \cos ^{2} l+\sin ^{2} l}{\left(\frac{a^{4}}{b^{4}} \cos ^{2} l+\sin ^{2} l\right)^{\frac{1}{2}}} ;{ }^{80}$ and considering $\frac{a}{b} \mid$ constant the $\int_{0}^{\left(x^{\prime 2}+y^{\prime 2}\right)^{1 / 2}} R^{\prime} \cos \cdot\left(\begin{array}{c}R^{\prime} \\ x^{\prime 2}\end{array}+y^{\prime 2}\right)^{1 / 2} d\left(x^{\prime 2}+y^{\prime 2}\right)^{1 / 2}=$ $\int_{0}^{b} \frac{4 \pi a^{2} b \delta k_{\prime}}{c^{3}}\left(\frac{c}{b}-\tan ^{-2} \frac{c}{b}\right) b d b=\frac{4 \pi a^{2} b \delta k^{\prime}}{c^{3}}\left(\frac{c}{b}-\tan ^{-1} \frac{c}{b}\right) \times \frac{1}{2} b^{2}, \mid$ (12) which is the sum of the components in the direction of the center of $R^{\prime}$ for all points on any line from the center| to the surface. Hence the weights of all columns from the center to the surface are equal, and by hypothesis the resultant of all forces on any part of the surface is perpendicular to it, therefore the fluid is in equilibrium at its| surface. And since the attraction of an ellipsoidal shell having its surfaces similar, concentric, and similarly placed,| is zero for an interior point, all the points of the homogeneous fluid ellipsoid are in equilibrium. And since it is found, by tracing the curve represented by (11.), when $b \div a$ and $\delta k$, are its co-ordinates, that $b \div a$ has only two values, it follows that there are only states of the equilibrium.

Second, When not of homogeneous density, let the ellipsoid of homogeneous density $\delta$ and semi axes $a$ and $b \mid$ be surrounded by a shell of density $\delta^{\prime}$, having the semi axes of its outer surface $a$, and $b$, coincident with $a$ and $b \mid$ throughout their extent, and proportional to them. Then the attraction of the ellipsoid and shell for any point| will be equal to the attraction of an 
ellipsoid whose density is $\delta^{\prime}$ plus or minus the attraction of one whose density $=\frac{1}{2} \delta-$ $\delta^{\prime}$, according as $\delta^{\prime}$ is less or greater than $\delta$. And the formula corresponding to (10.) is|

$$
R^{\prime}=\frac{\frac{4 \pi a_{\prime}{ }^{2} b_{l} \delta^{\prime} k_{\prime}}{c^{3}}\left(\frac{c_{\prime}}{b_{\prime}}-\tan ^{-1} \frac{c_{\prime}}{b_{l}}\right) \pm \frac{4 \pi a^{2} b\left(\delta-\delta^{\prime}\right) k_{\prime}}{c^{3}}\left(\frac{c}{b_{\prime}}-\tan ^{-1} \frac{c}{b_{\prime}}\right) b_{\prime}^{2}}{\left(a^{2} \cos ^{2} l+b^{2} \operatorname{sine}^{2} l\right)^{1 / 2}},(13)^{81} \text {. And the equilibrium of the }
$$

column| is proved as for the ellipsoid of homogeneous density. From (10.) or (13.) $\frac{R^{\prime}}{R^{\prime \prime}}=$ $\left(\frac{a^{2} \cos ^{2} l^{\prime \prime}+b^{2} \operatorname{sine}^{2} l^{\prime \prime}}{a^{2} \cos ^{2} l^{\prime}+b^{2} \operatorname{sine}^{2} l^{\prime}}\right)^{\frac{1}{2}}$

Hence $\frac{b^{2}}{a^{2}}=-\frac{1-\frac{R^{\prime 2}}{R^{\prime \prime 2}}}{\operatorname{sine}^{2} l^{\prime \prime}-\frac{R^{\prime 2}}{R^{\prime \prime 2}} \operatorname{sine}^{2} l^{\prime}}+1$, (15.) which is the square of the ratio semi axes ${ }^{82}$ of a rotating fluid mass| of any law of density, when in equilibrium.|

Since $R^{\prime} \cos .\left(\begin{array}{l}R^{\prime} \\ x^{\prime 2}\end{array}+y^{\prime 2}\right)^{1 / 2} d\left(x^{\prime 2}+y^{\prime 2}\right)^{1 / 2}=R^{\prime \prime} \cos .\left(\begin{array}{l}R^{\prime \prime} \\ x^{\prime 2}\end{array}+y^{\prime 2}\right)^{1 / 2} d\left(x^{\prime \prime 2}+y^{\prime \prime 2}\right)^{1 / 2}$, and $d\left(x^{\prime 2}+y^{\prime 2}\right)^{1 / 2}: d\left(x^{\prime \prime 2}+y^{\prime \prime 2}\right)^{1 / 2} \mid::\left(x^{\prime 2}+y^{\prime 2}\right)^{1 / 2}:\left(x^{\prime \prime 2}+y^{\prime \prime 2}\right)^{1 / 2}$, it follows that $R^{\prime} \cos .\left(\begin{array}{c}R^{\prime} \\ x^{\prime 2}\end{array}+y^{\prime 2}\right)^{1 / 2}\left(x^{\prime 2}+y^{\prime 2}\right)=R^{\prime \prime} \cos .\left(\begin{array}{l}R^{\prime \prime} \\ x^{\prime 2}\end{array}+y^{\prime 2}\right)^{1 / 2}\left(x^{\prime \prime 2}+y^{\prime \prime 2}\right), \mid$ (16.) showing the ${ }^{83}$ components toward the center at any two points on any surface similar to outside surface, are as| the distances from the center. ${ }^{84}$

If $n$ and $n^{\prime}$ are the lengths of arcs of the meridian, whose extremities differ in latitude by a small quantity, $\mid$ say $1^{\circ}, \frac{b^{2}}{a^{2}}=1-e^{2}=1-\frac{n^{2 / 3}-n^{\prime 2 / 3}}{n^{2 / 3} \operatorname{sine}^{2} l^{\prime \prime}-n^{\prime \frac{2}{3}} \operatorname{sine}^{2} l^{\prime}}$ which compared with (15.) shows that the lengths of the seconds| pendulum are as the cube roots of the arcs of a degree, when the figure is that due to a rotating fluid mass.|

The value of $\delta k_{\text {, }}$, from (11.) substituted in (13.), ${ }^{85}$ dividing both sides by $a \alpha^{2}$ the equatorial centrifugal force, $\mid$ and making $l=0$, and denoting by $R^{\prime} e$ the equatorial force of gravity, there results $\left|\frac{R^{\prime} e}{a \alpha^{2}}=\frac{\tan ^{-1} \frac{c}{b}-3 \frac{b c}{a^{2}}+2 \frac{b^{2}}{a^{2}} \tan ^{-1} \frac{c}{b}}{\tan ^{-1} \frac{c}{b}-\frac{b c}{a^{2}}}\right| 8687$

The first member, from the equatorial radius, and the length of the equatorial seconds pendulum as given by Prof. Airy, equals 289| nearly. Then solving the equation for $a \div b, 1-$ $a \div b$ the ellip-|ticity equals about $\frac{1}{215}^{88}$ instead of $\frac{1}{230}$ and $\frac{1}{300}$, the results usually| given. ${ }^{89}$

\section{COMMENTS ON GRAVITATION TO THE SPHERE}

Adcock's work is initially difficult to understand because it lacks an introduction, summary, and final conclusions (abstracts appear to have been rarely used in scientific journal articles before 1895). ${ }^{90}$ What is more, it contains no in-depth study nor comparison with existing work. It appears to be the culmination of several contributions by him on gravitational attraction and the flattening of the Earth, which he reported on at scientific meetings as mentioned, for example, in his paper Equilibrium of a fluid mass in the form of an ellipsoid rotating about its shorter axis. ${ }^{91}$ He seems to have considered the pamphlet transcribed here, which he refers to as 'my work on Gravitation' 92 to be his principal legacy to science, otherwise it cannot be understood why he went to the trouble of publishing it at his own expense and announcing it wherever he might find an open ear. 
In what follows we consider his findings and comment on each page of his pamphlet. In particular, we note what was known in his day and what his new contributions really were.

\section{PAGES 1 AND 2}

The only information as to the subject of his paper is given in its title. It concerns first, the gravitational force between two masses of ellipsoidal shape; and, second, the ratio of the axes of a rotating fluid body which is also ellipsoidal or, more precisely, a spheroid as a result of its rotation about an axis. The latter problem concerns the 'flattening' of a celestial object, such as Jupiter or the Earth.

The historical development of how to compute the gravitational interaction between spherical objects, and how to predict the flattening of rotating bodies mathematically, is fully described in the monograph by Todhunter. ${ }^{93}$ Such flattening has also been extensively analyzed in the book by Klein and Sommerfeld, ${ }^{94}$ in which many references can also be found.

However, finding explicit formulae which describe the gravitational interaction between ellipsoidal distributions of mass is more difficult. The dissertation by Schmidt provides a clue. $^{95}$ Heymann $^{96}$ states that Newton had attempted to find the gravitational field of a homogeneous ellipsoidal body but did not succeed, and that much later Laplace ${ }^{97}$ reconsidered the problem. Furthermore, although Laplace's method was complex, it was not strict enough and had to be improved later by Gauss and Dirichlet as part of their development of a general potential theory. ${ }^{98}$

Although, in principle, Adcock could have had access to these publications, there is no evidence that he was familiar with them other than the fact that, in a footnote, he states: 'my work on Gravitation ... will ... contain the only correct formula ever published for determining the figure of the earth on the hypothesis of fluidity, whether that figure be an ellipsoid of three unequal axes or two'. ${ }^{99}$ In a later comment, made in reply to a critic of his pamphlet and quoted in Section 5 (below), it appears that when his interest in this topic began, he was only familiar with the works of Airy, Pierce, Todhunter and Pratt. ${ }^{100}$

We believe that his findings were a truly original discovery which have become lost in time. Particularly so because, many years after his death, Otto Heymann, an engineer working at Siemens Apparate und Maschinen Gesellschaft in Berlin, also gave formulae for the potentials for an ellipsoid, apparently unaware of Adcock and his work. ${ }^{101}$

\section{PAGE 3}

Adcock's exposition begins with an introduction to his cumbersome nomenclature and then turns to analyzing the gravitational attraction in, and outside of, a (homogeneous) sphere, as well as a spherical shell. His arguments are quite unwieldy and lack the elegance of modern potential theory and vector calculus. ${ }^{102}$ Nevertheless, his final results for this case are correct: To an outside (unit) test mass, the gravitational action of the sphere or spherical shell is that of a mass of equivalent size situated in the center, and within a spherical shell there is no attraction

at all. However, these initial results are not new, as they can be found in Newton's Principia ${ }^{103}$ or in Maupertuis' work. ${ }^{104}$ 
Then follows the computation of the attraction by a prolate ellipsoid and an ellipsoidal shell, where the test mass is situated at a distance $h$ from the center of the major and minor axes, respectively. Adcock's results are confirmed by the formulae derived in the modern work of Heymann and Schmidt. ${ }^{105}$ Earlier versions of the same kind of analysis were given by Maclaurin, D'Alembert, Legendre and by Laplace ${ }^{106}$ Suffice it to say that their work also was not easy reading. As Todhunter remarked: 'Legendre's treatment of this particular case is sound but very laborious; he leaves much work to be effected by the reader, the results being given, but many of the intermediate operations being omitted. . . The student will find this result is correct, but the verification will be tedious'. ${ }^{107}$ Unfortunately Adcock's analysis and arguments on ellipsoidal attraction are not much easier to follow, even for those with some background in this kind of problem. Nevertheless he seems to have been one of the very first to give an explicit answer to the problem.

\section{PAGE 4}

The analysis of the prolate ellipsoid and ellipsoidal shell is completed and is then followed by an analogous one for the oblate case. As noted above, when Adcock uses the symbol 'log.', for example in Eqn. (7), he actually means the natural logarithm (i.e., 'ln.'). It should also be noted that the integration for the prolate case leading to Eqn. (7) can be converted into the final result for the oblate one, Eq. (12), by making use of the identity:

$$
\arctan z=\frac{1}{2 i} \ln \frac{1+i z}{1-i z} \text { with } z=\frac{\sqrt{a^{2}-c^{2}}}{r} .
$$

By using expressions such as 'sine ${ }_{x}^{R}$ ', Adcock seems to have adopted a nomenclature similar to that used by Peirce in which: '. $\ldots \begin{aligned} & p p p \\ & x^{\prime} y^{\prime} z^{\prime}\end{aligned}$ denote the angles which this motion makes with the three mutually perpendicular axes, called the axes of $x, y$, and $z, \ldots, 108$

\section{PAGE 5}

Adcock starts this page with a passage on the attraction between shells. The reason for this is probably because only the attraction of a point-like test mass by ellipsoidal mass distribution had been considered so far. He then prepares the reader for the notion of gravitational potential (see Eqn. 18). Because the mathematical power of vector calculus was not known to him, as Wilson and Gibbs (1901) textbook ${ }^{102}$ had not yet been published, this is achieved in a very crude way which can only be generalized to a general three-dimensional case (which he will need later) with great effort. Moreover, from today's didactic point of view, it is also questionable why Adcock did not begin his exposition with the notion of gravitational potential for an ellipsoidal mass distribution. As a matter of fact, the potential within, and outside of, an arbitrary mass distribution can be found by solving the Poisson equation, which was published over 50 years earlier. ${ }^{109}$ It seems that this equation, and the corresponding more abstract and fundamental meaning of gravitational potential, were unknown to Adcock, because he starts to derive an expression for it from the more intuitive integral expressions for forces, which he relates to the notion of work: 'Let the term potential $=\omega$, be the general integral of the 
elementary quantity of work of forces directed to and dependent on the distances to fixed centers from the attracted point.'

\section{PAGE 6}

This deals with the integration process involved in determining the attraction of prolate ellipsoids for an arbitrary external point (in contrast to the previous situation, where it was confined to a position along the plane through the equator). It ends with an explicit relation for the force component perpendicular to the major axis.

Note Adcock's use of single and double colons in $c^{2}: c^{2}-x^{2}:: \frac{a b^{2} \delta}{c}: \frac{a b^{2} \delta\left(c^{2}-x^{2}\right)}{c^{3}}$ (for example). The meaning of this notation, which was commonly in use in Europe from the mid18th century onwards, and in the United States until the beginning of the 20th century, is as follows: ' $\mathrm{A}: \mathrm{B}=$ the ratio of $\mathrm{A}$ to $\mathrm{B}$; and $\mathrm{A}: \mathrm{B}:: \mathrm{C}: \mathrm{D}=$ the separation of two equal ratios, e.g., $3: 12:: 2: 8$.' 110

\section{PAGE 7}

Adcock begins by concluding his analysis from Page 6, and finally presents the formula for the component parallel to the major axis of the prolate ellipsoid. A similar analysis is then performed for the case of an oblate ellipsoid. In fact, both cases could have been combined by using the relationship relating arctan to $\ln$ ( $c f$. Comments, Page 4, above).

He finally turns to the problem of a rotating fluid mass in order to determine the degree of flattening of self-gravitating bodies. He first shows that the rotation about an axis results in an oblate ellipsoid of revolution, which he calls the equilibrium shape. Alternatively, he could have said that this is the shape assumed by an incompressible self-gravitating rotating body after a stationary state has been reached. His analysis seems clumsy in comparison with today's standards based on string-end continuum mechanics. ${ }^{11}$

\section{PAGE 8}

Finally, Adcock gives an expression which allows him to calculate a value for the flattening:

$f=1-\frac{1}{\lambda}, \lambda=\frac{a}{b}>1$, where $a$ is the equatorial axis and $b$ the polar axis of the rotating spheroid. Of paramount importance in the calculation is the value 289 taken for the ratio between the gravitational and the centrifugal acceleration at the equator, as given in a paper by Airy ${ }^{112}$ :

$$
\frac{m /\left(G a^{2}\right)}{a \omega^{2}}=289,
$$

where $m$ denotes the mass of the fluid mass subjected to self-gravitation, rotating at an angular speed $\omega$; and $G$ is the universal constant of gravitation. It serves as input on the left hand side 
of Adcock's last formulae on this page. In fact, writing Adcock's formulation in modern terminology:

$$
\frac{R_{e}^{\prime}}{a \alpha^{2}} \equiv \frac{m /\left(G a^{2}\right)}{a \omega^{2}} .
$$

Note that his original equation is faulty and should be replaced by the final equation in his errata list. ${ }^{86}$ Its subsequent evaluation can only be performed numerically. If evaluated using a modern software tool such as Mathematica, ${ }^{113}$ the flattening comes out to be $1 / 232.186$, which agrees exactly with the value given in the last item in Adcock's list of errata. ${ }^{88}$ Comparison with the other two estimates given in the penultimate line of his corrections suggests that he must have obtained it by means of an iterative calculation.

Is his result for the flattening really physically significant? Consider the simple formula originally obtained by Newton, who found the ratio between the gravitational and the centrifugal acceleration to be: ${ }^{114}$

$$
\frac{m /\left(G a^{2}\right)}{a \omega^{2}}=\frac{5}{4 f} .
$$

By replacing the left hand side of this expression, the ratio between the gravitational and the centrifugal acceleration at the equator, by Airy's experimentally-determined value 289, one obtains $f=\frac{5}{4 \times 289}=\frac{1}{231.2}$, which is not much different from Adcock's result. Moreover, if the theoretical result derived by Müller: ${ }^{29}$

$$
\frac{m /\left(G a^{2}\right)}{a \omega^{2}}=\frac{3}{2} \frac{\left(1+2 \lambda^{2}\right) \arccos (\lambda)-3 \lambda \sqrt{1-\lambda^{2}}}{\left(1-\lambda^{2}\right)^{\frac{3}{2}}},
$$

is evaluated numerically, one finds $f=\frac{1}{231.99}$ and comes somewhat closer to Adcock's value. However, experimental findings indicate that the true flattening value for planet Earth is $f=\frac{1}{298.24}$ (Fig. 1). ${ }^{33}$ In other words, the Earth looks much less like a 'jelly donut' than is predicted from the fluid model. It is stiffer because it also contains solid layers and, in fact, this makes the whole controversy as to which value is correct a purely academic argument.

\section{A CONTEMPORARY CRITIC}

Anonymous criticism of Adcock's work, presumably by an early purchaser of his pamphlet, appeared in the Chicago tribune (30 March 1872, p. 4):

It has long been believed and taught that the flattening of the earth in the polar regions is due to the fact that the force of gravity at the equator is partially counteracted by the centrifugal force generated by the daily rotation of the earth on her axis. It is also held that this flattening, which amounts to about one part in 300, shows that the centrifugal force of the equator is equal to about one part in 289 of the force of attraction there. 
Mr. R.J. Adcock, of Monmouth, Ill. has written a pamphlet, the object of which is to prove that these conclusions are erroneous. He shows, by a formidable array of quantities, stated in the language of the Calculus, that the ratio of these two forces is between 373.12 and 373.76 instead of $289,{ }^{115}$ and that, therefore, either the earth has not the form due to a rotating fluid mass, or that the lengths of its equatorial and polar diameters have not been ascertained.

The comment in the first paragraph above regarding centrifugal force refers to Newton's deduction (see above) that: $\lambda=\frac{1}{289}$. Adcock replied in a letter to the same newspaper (15 April, 1872, p. 5):

I have to say it is definitely known ... that, the form of the surface of the earth not being that of an exact water-level of equilibrium, the tendency of gravity, including centrifugal force, together with the dissolving effects of the fluids on the surface, is to produce that exact form of equilibrium of the surface; and, that the equatorial diameter of this surface of equilibrium will be greater, other things being the same, with centrifugal force than it would be without rotation. Then the flattening, or a part of it, is either due to, or maintained or increased by, rotation, according as the surface differs from one of equilibrium.

The one part in 300 , and the one part in 289 , are both deduced by correct methods from actual observation and measurement, and therefore I do not undertake to dispute these results. What I have proved is, that the so universally affirmed proposition, that the agreement of these two results with the hypothesis that the earth has the approximate form due to fluidity, is not the case.

Your conclusion that the flattening of about one part in 300 shows a ratio of one part in 289 of the centrifugal force to gravity, is certainly very wild: since, except in the case of fluidity, the rotation between the flattening and this ratio could not be determined theoretically, even in the case of the perfect equilibrium of the surface, without knowing the internal constitution of the earth. And, in the case of perfect fluidity, I have shown - from formulae . . that this flattening of one part in 300 , and the ratio of 289 , are widely inconsistent with the condition of fluidity.

Now, as to my object in writing the treatise: It is to show for the benefit of science and myself, the accomplishment of what is mentioned in the title-page, to find the "Attraction to the sphere and the two ellipsoids of revolution," and to find the "ratio of the axes of a rotating fluid mass" under the action of the gravity of its own particles, according to the universal law of Newton.

When I adopted my method of treating attraction, I was acquainted only with that given in Professor Pierce's [sic.] Analytical [sic.] Mechanics, ${ }^{116}$ which I thought might be rendered more elementary; and, after examination of Professor [I.] Todhunter's, J.H. Pratt's and Professor Airy's methods, I considered mine, if not shorter, at least more easily understood, original to some extent, and American, accomplishing by direct integration what J.H. Pratt said was "impossible to integrate by any known method of direct integration". ${ }^{117}$

And, after correctly solving the problem of the form and ratio of the axes of a rotating fluid mass under the action of the gravity of its own particles, I was convinced that this problem, though attempted by Newton, and held since [Clairaut] 1743 to have been solved by Clairaut, Jacobi, Laplace, Bowditch, Airy, ${ }^{118}$ etc., etc., was correctly solved for the first time, though I was not able then, and not until quite recently, to show what was the error in the reasoning of others on this subject, which is simply in violating the axiom that, in the same calculation, it will not do to use as equal two different units of measure of the same or similar quantities ... [these] are two differing measuring units of force, - one the attraction of a unit of mass at the unit of distance, the other the centrifugal force of a unit of mass revolving with a unit of velocity in a circle of radius unity.

Adcock completed the arguments for his proof that 'a fluid mass, in the form of an ellipsoid, rotating about its shortest axis, under the action of the attraction of its own particles and their centrifugal forces, is in equilibrium; and this is the only form of equilibrium' in a short note (which contains no numerical results) published in December of that year. ${ }^{19}$ However, his publications on the topic appear not to have attracted attention subsequently. It is probable that his pamphlet appeared too late to be included in Todhunter's definitive survey of publications on 'the figure of the earth' (since its preface is dated July of 1873) ${ }^{31}$ In any case, by that time 
interest in the problem had essentially lapsed. His pamphlet became effectively lost thereafter and is transcribed here for the first time. See Müller (2018) for a modern view of the subject. ${ }^{29}$

\section{CONCLUSIONS}

Adcock's (1872) paper on the figure of the earth gives a complete, if somewhat crude, derivation of the solution of a problem first considered, but not solved, by Newton, Laplace, Gauss and Dirichlet: namely, finding the gravitational field of a rotating self-gravitating homogeneous ellipsoidal fluid mass. After lengthy calculations, all the more remarkable as they were undertaken without the benefits of potential theory and vector algebra, he obtained a formula which, until now, was believed to have first been derived by Heymann (1935). Using Airy's (1856) value for the ratio between the gravitational and centrifugal acceleration at the equator, Adcock obtained a value for the inverse flattening of 232.19, which is very close to the 'exact' value of 231.99, obtained using modern analytic techniques applied to a model of a self-gravitating sphere made of an incompressible fluid which starts spinning about a fixed axis at a constant angular velocity and which, a result of centrifugal accelerations and internal friction, finally reaches a state of axisymmetric deformation in which it assumes the shape of a spheroid. ${ }^{29}$ The numerical value Adcock hand-calculated for the flattening achieved under the model is extremely close to that obtained using Wolfram's Mathematica software today. Curiously, he concluded his paper with the comment: 'the ellipticity equals between $\frac{1}{232.016}$ and $\frac{1}{232.698}$ instead of $\frac{1}{230}$ and $\frac{1}{300}$ the results usually given' without pointing out that his work applied to an entirely theoretical model, and that the difference between his result and the real-world observed value of 298.24 for the Earth is accounted for by the fact that the latter has a more complex interior structure.

\section{ACKNOWLEDGEMENT}

We would like to thank: Rupert Baker, Library Manager, The Royal Society and his staff for their for their interest and assistance with our investigation.

\section{NOTES}

$1 \quad$ Robert J. Adcock, 'The Correct Method of Least Squares', The Analyst, 1, 64 (1874); 'Note on the method of least squares', ibid., 4, 183-184 (1877); 'Least squares', ibid., 5, 21-22 (1878a); 'A problem in least squares', ibid. 5, 53-54 (1878b); 'Extension of the method of least squares to any number of variables', ibid., 7, 22-23 (1880).

Adcock, op. cit. (note 1) $1878 b$, equation 5 at p. 54.

Adcock, 'Solution' [to Problem 239]. The Analyst, 6, 49 (1879).

Robert Hooke, An Attempt to Prove the Motion of the Earth from Observations. (J. Martyn.

London, 1674) at pp. 27-28.

Ibid. at p. 28. 
The letter is reproduced as document 239 in The correspondence of Isaac Newton. Vol. 2. 16761687. (eds. Hubert WestrenTurnbull, J.F. Scott, A. Rupert Hall and Laura Tilling). (Cambridge University Press, Cambridge, 1960). at p. 309.

See Michael Nauenberg, 'Robert Hooke's seminal contribution to orbital dynamics'. Physics in Perspective, 7, 4-34. (2005).

8 Isaac Newton, I. Bernard Cohen, Anne Whitman \& Julia Budenz. Isaac Newton. The Principia. Mathematical Principles of Natural Philosophy. A new translation. Preceded by A guide to Newton's Principia by I. Bernard Cohen (University of California Press, Berkeley \& London, 1999) at pp. 595-596); see also: Isaac Newton, Philosophiae naturalis principia mathematica (G. \& J. Innys, London, 1687); The Mathematical Principles of Natural Philosophy. Translated into English by Andrew Motte. To which are added, The Laws of the Moon's Motion according to Gravity By John Machin. 2 vols. as one. (Benjamin Motte, London, 1729), at II, pp. 272-274. Subrahmanyan Chandrasekhar, Newton's Principia for the Common Reader (Clarendon Press, Oxford, 1995) at pp. 280-282.

10 Pierre Bouguer, La Figure de la Terre, Déterminée par les Observations de Messieurs Bouguer et de la Condamine ... pour observer aux environs de l'Equateur. Avec une Relation abregée de ce Voyage, qui contient la description du Pays dans lequel les Opérations ont été faites (Charles-Antoine Jombert, Paris, 1749).

11 Neville Maskelyne, 'An Account of Observations made on the Mountain Schehallien for finding its Attraction'. Philosophical Transactions of the Royal Society, London, 65, 500-542 (1775); see also John R. Smallwood, 'Maskelyne's 1774 Schiehallion experiment revisited'. Scottish Journal of Geology, 43, 15-31 (2007). Henry Cavendish, 'Experiments to Determine the Density of the Earth'. Philosophical Transactions of the Royal Society, London, 88, 469-526 (1798).

13 David W. Hughes, 'The mean density of the Earth'. Journal of the British Astronomical Association, 116, 21-24 (2006).

14 Charles Vernon Boys, 'On the Newtonian constant of gravitation'. Philosophical Transactions of the Royal Society, London, Series A, 186, 1-72 (1895); 'The Newtonian constant of gravitation'. Notices of the proceedings at the meetings of the members of the Royal Institution of Great Britain, 14, 353-377 (1896).

15 However, $G$ has proved extremely difficult to measure precisely and even the current value, $6.67408 \times 10-{ }^{11} \mathrm{~m}^{3} \mathrm{~kg}^{-1} \mathrm{~s}^{-2}$, established in 2014 by the International Council for Science Committee on Data for Science and Technology (James E. Faller, J.E. 2014. 'Precision measurement, scientific personalities and error budgets: the sine quibus non for big G determinations'. Philosophical Transactions of the Royal Society, London, Series A, 372, no. 2026 (2014) (http://dx.doi.org/10.1098/rsta.2014.0023)) has an uncertainty of $0.00031 \times 10^{-11}$ (National Institute of Standards and Technology. The NIST reference on constants, units and uncertainty. Fundamental physical constants. Newtonian constant of gravitation. (2018) (https://physics.nist.gov/cgi-bin/cuu/Value?bg. 2018).

16 Chandrasekhar, op. cit. (note 9) at p. 373.

17 Ibid. See also: John Henry Poynting, The mean density of the Earth. An essay to which the Adams Prize was adjudged in 1893 in the University of Cambridge. Charles Griffin, London (1894); Keith Edward Bullen, The Earth's density. Chapman \& Hall, London (1975); and Hughes, op. cit. (note 13), for further discussion. Newton, op. cit. (note 8), II, at. p. 245.

Ibid. Its diameters are : polar 81,430 mi (131,049 km); equatorial 86,519 $\mathrm{mi}(139,239 \mathrm{~km})$. An archaic measure: 12 Paris lines $=1$ Paris inch; 12 Paris inches $=1$ Paris foot $(32.48406 \mathrm{~cm})$; 6 Paris feet $=1$ Paris fathom (toise). Martin, J.-B. Coignard \& H.-L. Guerin, Paris. pp. 176-178 (1733) at p. 7. 
Andrew Motte's 1729 translation (note 8) was based on Newton's second (1713) and third (1726) Latin editions; see also: John Martin Frederick Wright. Commentary on Newton's Principia with a supplementary volume. Designed for the use of students at the universities. I. Black, Young and Young, London. (1828). See Giuseppe Iurato, 'The dawning of the theory of equilibrium figures: a brief historical account from the 16th through the 20th century' (2014) (https://arxiv.org/abs/1409.3858v2) for a history of the concept of an equilibrium figure. Newton, op.cit. (note 8), at pp. 230-231.

Ibid. at pp. 240-241.

Ibid. at p. 243.

John L. Greenberg, The problem of the Earth's shape from Newton to Clairaut. The rise of mathematical science in eighteenth-century Paris and the fall of "normal" science. Cambridge University Press, Cambridge (1995), at p. 7. Chandrasekhar, op. cit. (note 9) at pp. 384-392. Wolfgang $\mathrm{H}$. Müller, The effect of rotation on the flattening of celestial bodies: A journey through four centuries. Mathematics and Mechanics of Complex Systems. 6, 1-40 (2018) (doi: dx.doi.org/10.2140/memocs.2018.6.1).

Newton, op. cit., (note 8), at p. 250.

31 Alexis-Claude Clairaut, Théorie de la figure de la terre, tirée des principes de l'hydrostatique. David Fils, Paris (1743); Adrien-Marie Legendre, Suite des recherches sur la figure des planètes. Histoire de l'Académie des Sciences. Année MDCCLXXXIX. Avec les Mémoirés de Mathématique et de Physique, pour la même année. Tirés des registres de cette Académie. [Paris] (1793), pp. 372-454; Colin Maclaurin, A Treatise on Fluxions. In two volumes. Second edition. To which is prefixed an account of his life. William Baynes \& William Davis. London. (1801); Pierre-Simon Laplace, Traité de Mécanique Céleste. Tome Cinquième. Bachelier, Paris. (1823). See also: Pierre Simon Laplace, Mécanique céleste (trans. Nathaniel Bowditch). Vols. 1-4. (Isaac R. Butts \& Co.; Charles C. Little \& James Brown; Hilliard, Gray \& Co.; Boston, 1829-39); Greenberg, op. cit. (note 27) and Isaac Todhunter, A history of the mathematical theories of attraction and the figure of the earth. From the time of Newton to that of Laplace. (vols. I and II, as one). Macmillan, London. (1873), for discussion. Legendre, op. cit. (note 31), at p. 411. The currently accepted value for the mean density of the whole Earth is $5.52 \mathrm{gm} \mathrm{cm}^{-3}$; and that of the core, which occupies the innermost $55 \%$, is 16.9 $\mathrm{gm} \mathrm{cm}^{-3}$. See: Adam M. Dziewonski and Don L. Anderson. Preliminary reference Earth model. Physics of the Earth and Planetary Interiors. 25, 297-356 (1981).

Data from Newton, op. cit. (note 8), Todhunter, op.cit. (note 31) and Georg Straßer, Ellipsoidische parameter der Erdfigur (1800-1950). Deutsche Geodätische Kommission bei der Bayerischen Akademie der Wissenschaften. Reihe A: Höhere Geodäsie, 19. (Bayerischen Akademie der Wissenschaften, Munich, 1957) and David Richard Williams, Planetary fact sheet: metric. National Aeronautics and Space Administration (2018). (http://nssdc.gsfc.nasa.gov/planetary/factsheet). John Henry Pratt, A Treatise on Attractions, Laplace's functions, and the Figure of the Earth. (Macmillan, London, 1860). George Biddell Airy, 'Account of pendulum experiments undertaken in the Harton Colliery, for the purpose of determining the mean density of the earth. \& ... Supplement to the "Account of pendulum experiments undertaken in the Harton Colliery;" being an account of experiments undertaken to determine the correction for the temperature of the pendulum'. Philosophical Transactions of the Royal Society, London. 146, 297-355 (1856). Pratt, op. cit. (note 34), at p. 79.

Ibid. at p. 65.

Chicago tribune, 30 Mar. 1872, at p. 4 (https://chroniclingamerica.loc.gov/search/titles/). Based on the Consumer Price Index (https://www.measuringworth.com/calculators/uscompare/ relativevalue.php). 
In the copy in the library of The Royal Society, London, both 'Galesburg' and 'Utah' are crossed through in pencil and changed to 'Monmouth'. The same text also appears as an advertisement in The Journal of the Franklin Institute, Philadelphia, 113 (for 1872) at pp. 216, 362 and xxiii, but with Monmouth substituted. Unnumbered page in the original.

RJA: for center read centre, and at all other places where center occurs.

The figure on this page is unnumbered in the original.

The $n$ in the upper bound of the first integral is most likely confused with the letter $u$.

RJA: after $C e$ and before (3.), insert a comma.

The integration should be with respect to $d \theta$ not $d a$. There is a minus sign missing after the integration is carried out. However, this does not matter if the upper and lower bounds are inserted. The final result after the integration is correct.

RJA: eq. (5.), for $\tan ^{2} \theta$ read $\operatorname{sine}^{2} \theta$.

RJA: for tan. ${ }^{-1} \frac{b}{h^{2}-a^{2}}$ read tan..$^{-1} \frac{b}{\left(h^{2}-a^{2}\right)^{1 / 2}}$.

RJA: for $a^{2} b$ read $a b^{2}$; note that where Adcock uses the symbol "log.," he actually means the natural logarithm, i.e. "In."

RJA: insert before (7.), ).

"with respect $c$ " should read "with respect to $c$."

RJA: for $\pi$ read $\frac{1}{2} \pi$.

"infinitessimal" should read "infinitesimal."

RJA: for $2 x^{\prime} y^{\prime}$ read $2 \alpha x y^{\prime}$.

RJA: for $2 x^{\prime} y^{\prime}$ read $2 \alpha x y^{\prime}$.

RJA: for $1+\frac{\beta \mp \delta}{1 \pm \beta \delta} \operatorname{read} 1+\left(\frac{\beta \mp \delta}{1 \pm \beta \delta}\right)^{2}$; for $\alpha^{2}$ read $a^{2}$.

RJA: read for the first power of $s$ read odd powers of $\delta$.

RJA: for 2 read 3.

RJA: for $b^{2} c^{2}$ cos. $^{2} u$ read $b_{l}^{2} c^{2} \cos ^{2} u$.

RJA: for 6 read 4; and for these read then.

RJA: the sign before $c$, in the numerators of the $2 \mathrm{~d}$ and 5 th terms, to be changed.

RJA: for $\left(a_{r}^{4} y^{\prime 2}+b_{l}^{4} x^{\prime 2}\right)^{1 / 2} \frac{d x^{\prime}}{d y^{\prime}} \operatorname{read}\left(a_{1}^{4} y^{\prime 2}+b_{l}^{4} x^{\prime 2}\right)^{1 / 2} \frac{d x^{\prime}}{b_{l}^{2} x^{\prime}}$.

RJA: after $\frac{b_{1}}{a}$, and before $a^{\prime 2}$, insert (.

RJA: for $d^{\prime}$ read $d x^{\prime}$; and in the denominator, for $c^{2}$ read $x^{\prime 2}$.

RJA: for $y^{\prime 4}$ read $y^{\prime 2}$.

RJA: for $\frac{a_{\prime} y^{\prime}}{\left.a_{I}^{2}-x^{\prime 2}\right)^{\frac{1}{2}}} \operatorname{read} \frac{a_{1} y^{\prime}}{\left(a_{l}^{2}-x^{\prime 2}\right)^{1 / 2}}$.

RJA: for $\frac{a_{1} c y^{\prime 2} d c}{a_{l}^{4}-c^{2} x^{\prime 2}} \operatorname{read} \frac{a_{l}^{3} c y^{\prime 2} d c}{b_{l}^{2}\left(a_{l}^{4}-c^{2} x^{\prime 2}\right)}$.

RJA: for $y$ read $y^{\prime}$.

RJA: for $\left.a_{t}^{2}-x^{\prime 2}\right)^{1 / 2} \mathrm{read}\left(a_{r}^{2}-x^{\prime 2}\right)^{1 / 2}$.

"transeverse" should read "transverse."

RJA: for $b_{14}$ read $b_{1}^{4}$; and for $\left(a_{1}^{2}-x^{\prime 2}\right)^{3} \mathrm{read}\left(a_{1}^{2}-x^{\prime 2}\right)^{\frac{3}{2}}$.

RJA: eq. (2.) for $\operatorname{sine}_{x}^{R}$ read $\sin \underset{x}{R^{\prime}}$.

RJA: eq. (5.), put $b$ as a factor in the second member.

"parallell" should read "parallel."

RJA: eq. (10.), put $b^{2}$ as a factor in the second member.

RJA: And $\alpha^{2}$ as a factor in the last term of the next equation.

RJA: after results, read, by making $y^{\prime}=0$.

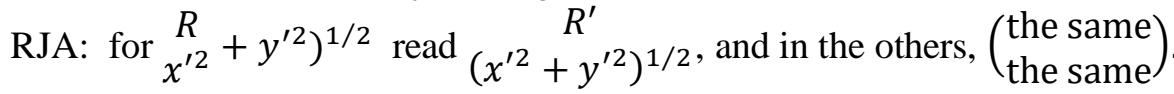


RJA: before $\frac{a^{2} \cos ^{2} l+\operatorname{sine}^{2} l}{\left(\frac{a^{4}}{b^{4}} \cos ^{2}{ }^{2} l+\operatorname{sine}^{2} l\right)^{1 / 2}}$ read $=$.

RJA: for $a^{2}$ read $a_{1}^{2}$; and for $b^{2}$ read $b_{r}^{2}$.

RJA: for ratio semi axes read ratio of the semi axes.

RJA: for showing the read showing that the.

RJA: for center read centre reciprocally.

RJA: for (13.) read (10.).

RJA: For last equation, read $\frac{R^{\prime}}{a \alpha^{2}}=\frac{2 b^{2}\left(\frac{c}{b}-\tan ^{-1} \frac{c}{b}\right)}{\left(a^{2}+2 b^{2}\right) \tan \cdot^{-1} \frac{c}{b}-3 b c}$, as in last line [N.B. $R^{\prime}$ here should read $\left.R_{e}^{\prime}.\right]$

In the original, this equation is inserted at the bottom-left of the page and the next three lines here are placed to its right.

RJA: In last line but one, for about $\frac{1}{215}$ read between $\frac{1}{232.016}$ and $\frac{1}{232.698}$; The fifth approximating fraction of $\tan ^{-1} \frac{c}{b}$ substituted in $\frac{R_{e}^{\prime}}{a \alpha^{2}}$, giving $\frac{1}{232.18}$.

Followed in the original by an unnumbered page consisting of 39 Errata, given here as footnotes.

Pontus Plavén-Sigray, Granville James Matheson, Björn Christian Schiffler and William Hedley Thompson, Research: The readability of scientific texts is decreasing over time. (2017). eLife 2017; 6:e27725. DOI: 10.7554/eLife.27725.

Robert J. Adcock, 'Equilibrium of a fluid mass in the form of an ellipsoid rotating about its shorter axis.' The American Journal of Science and Arts. Ser. 3, 4, 506 (1872).

Ibid., at p. 506, footnote.

Todhunter, op. cit. (note 31).

Felix Klein \& Arnold Sommerfeld, Über die Theorie des Kreisels. H. 3. Die störenden Einflüsse : astronomische und geophysikalische Anwendungen. (B.G. Teubner, Leipzig, 1903); Felix Klein \& Arnold Sommerfeld, (trans. Raymond J. Nagem \& Guido Sandri). The theory of the top, vol. III: Perturbations. Astronomical and Geophysical Applications. (Birkhäuser, Boston; Springer, London, 2012); . Maarten Schmidt, A model of the distribution of mass in the galactic system (Doctoral dissertation, Leiden University, 1956); 'A model of the distribution of mass in the galactic system'. Bulletin of the Astronomical Institutes of the Netherlands, 13 (468), 15-41 (1956). Otto Heymann, 'Über das Gravitationspotential eines homogenen Ellipsoids'. Astronomische Nachrichten, 256, 181-186 (1935).

Pierre-Simon Laplace, 'Théorie des attractions des sphéroides et de la figure des planètes'. Histoire de l'Académie Royale des Sciences. Année 1782. Avec les Mémoires de Mathématique \& de Physique, pour la même Année, Tirés des Registres de cette Académie. (Académie Royale des Sciences, Paris, 1785) at pp. 113-196.

Carl Friedrich Gauss, 'Theoria attractionis corporumm sphaeroidicorum ellipticorum homogeneorum'. Methodo nova tractata. Commentationes Societatis Regiae Scientiarum Gottingensis Recentiores. II. Classis Mathematicae. [paper 3], 1-24 (1813); Peter Gustav Lejeune Dirichlet, 'Über eine neue Methode zur Bestimmung vielfacher Integrale'. Mathematische Abhandlungen der Königlichen Akademie der Wissenschaften zu Berlin. Aus dem Jahre 1839. 61-79 (1841).

Robert J. Adcock, 'Equilibrium of a fluid mass in the form of an ellipsoid rotating about its shorter axis'. The American Journal of Science and Arts. Ser. 3, 4, 506 (1872).

George Biddell Airy, 'Figure of the Earth' in Encyclopaedia Metropolitana; or universal dictionary of knowledge, on an original plan: comprising the twofold advantage of a philosophical and an alphabetical arrangement (eds. Edward Smedley, Hugh James Rose and Henry John Rose). V, pp. 165-240 (B. Fellowes et al., London, 1845); Mathematical tracts on the lunar and planetary theories, the figure of the Earth, precession and nutation, the calculus 
of variations and the undulatory theory of optics. Designed for students in the University. 4th edn. (Macmillan, Cambridge, 1858); Benjamin Peirce, A system of analytic mechanics. (Little, Brown and Co., Boston, Massachusetts, 1855); probably Isaac Todhunter, A treatise on the integral calculus and its applications with numerous examples. (Macmillan, Cambridge, 1857); Pratt, op. cit. (note 34).

101 Heymann, op. cit. (note 96).

102 However, the latter only became common knowledge some years later, as a result of lectures given at Yale University in the 1880s by the American mathematical physicist, Josiah Willard Gibbs (1839-1903); Edwin Bidwell Wilson and Josiah Willard Gibbs. Vector analysis: A textbook for the use of students of mathematics and physics: founded upon the lectures of J.W. Gibbs. (Yale University Press, New Haven, Connecticut, 1901). Todhunter, op. cit. (note 31) at $§ 4 \& 5$ (I, pp. 2-3); see also: Chandrasekhar, op. cit., (note 9) at pp. 269-302).

104 Pierre Louis Moreau de Maupertuis, 'Sur la loix de l'attraction'. Mémoires de l'Academie Royale des Sciences. Année 1732. Paris. 343-362 (1735); see also: Todhunter, op. cit. (note 31) at $\S 128 ; \mathbf{I}$, p. 67.

105 Heymann, op.cit. (note 96); Schmidt, op. cit. (note 95) 1956 .

106 Colin Maclaurin, 'De Causa Physica Fluxus et Refluxus Maris' (1740) in (eds. Thomas Le Seur, \& François Jacquier) Philosophiae naturalis principia mathematica. Auctore Isaaco Newtono, eq. aurato. Perpetuis commentariis illustrata, communi studio. Barrillot \& Fillii, Geneva. III, 247-282 (1742); see also: Ian Tweddle, MacLaurin's Physical Dissertations. Sources and Studies in the History of Mathematics and Physical Sciences. (Springer-Verlag, London, 2007), at 103-127; Jean le Rond D’Alembert, 'XLVII Mémoire. Suite des Recherches sur la Figure de la Terre', in Opuscules Mathematiques, ou Mémoires sur différens Sujects de Géométrier, de Méchanique, d'Optique, d'Astronomie, \&c. Tome VI. (Briasson, Paris, 1773) at pp. 161-246; Adrien-Marie Le Gendre [Legendre], 'Suite des recherches sur la figure des planètes'. Histoire de l'Académie des Sciences. Année MDCCLXXXIX. Avec les Mémoirés de Mathématique et de Physique, pour la même année. Tirés des registres de cette Académie. (Libraires de l'Académie des Sciences, Paris, 1793) at pp. 372-454; Pierre-Simon de la Place [Laplace], Théorie du mouvement et de la figure elliptique des planètes. ( $\mathrm{Ph} .-\mathrm{D}$. Pierres, Paris, 1784); Laplace, P.-S. Traité de Mécanique Céleste. Tome Premier. (Crapelet, Paris, 1798); see also: Todhunter, op. cit. (note 31) at: $\S 254,625,805 \& 880$; I, pp. 139-140, 402 and II, pp. 32, 76.

107 Todhunter, op. cit. (note 31) at: II, p. 76.

108 Pierce, op.cit. (note 100) at p. 10.

109 Baron Siméon Denis Poisson, 'Remarques sur une équation qui se présente dans la théorie des attractions des sphéroïdes'. Nouveau Bulletin de la Société philomathique de Paris, 3, 388-392 (1812).

110 Florian Cajori, A history of mathematical notations. 1. Notations in elementary mathematics. (The Open Court Company, London, 1928) at p. 294.

111 See, for example, the arguments in the lecture notes by Richard Fitzpatrick: Fluid mechanics, lecture notes, University of Texas at Austin, 2016 (http://farside.ph.utexas.edu/teaching/ 336L/Fluid /.2016) at p. 45, which make it immediately obvious that the symmetric ellipsoid, namely a spheroid, is the stationary equilibrium shape for an incompressible fluid body subjected to rotation about an axis. Airy, op. cit. (note 35) at p. 355.

Wolfram Research, Campaign, Illinois; first released in 1988. Chandrasekhar, op.cit. (note 9) at p. 384.

It is not clear where the figures of $\sim 373$ in this correspondence come from. They are not in the printed copy of Adcock's pamphlet nor in any newspaper article or correspondence which it has been possible to trace, but the present-day collections of scanned newspapers do not, unfortunately, provide a complete record.

116 Peirce, Benjamin, A system of analytic mechanics. (Little, Brown. Boston, 1855). 
117 Inexact quotation from John Henry Pratt, $A$ Treatise on Attractions, Laplace's functions, and the Figure of the Earth. (Macmillan, London, 1860) at p.10.

118 Carl Gustav Jacobi, 'Ueber die figur des gleichgewichts'. Annalen der Physik, 109, 229-233 (1834).

119 Robert J. Adcock, 'Equilibrium of a fluid mass in the form of an ellipsoid rotating about its shorter axis'. The American Journal of Science and Arts. Ser. 3. 4, 506 (1872). 